\title{
The Exponentiated Burr XII Weibull Distribution: Model, Properties and Applications
}

\author{
Boikanyo Makubate $^{\mathrm{a}}$, Neo Dingalo ${ }^{\mathrm{a}}$, Broderick O. Oluyede ${ }^{\mathrm{b}}$, Adeniyi F.Fagbamigbe ${ }^{\mathrm{a}, \mathrm{c}}$
}

\begin{abstract}
${ }^{a}$ Department of Mathematics and Statistical Sciences, Botswana International University of Science and Technology, Palapye, BW ; ${ }^{b}$ Department of Mathematical Sciences, Georgia Southern University, Statesboro, GA, 30460,USA ; ${ }^{c}$ Department of Epidemiology and Medical Statistics, College of Medicine, University of Ibadan, $N G$
\end{abstract}

\begin{abstract}
A new distribution called the exponentiated Burr XII Weibull(EBW) distributions is proposed and presented. This distribution contains several new and known distributions such as exponentiated log-logistic Weibull, exponentiated log-logistic Rayleigh, exponentiated log-logistic exponential, exponentiated Lomax Weibull, exponentiated Lomax Rayleigh, exponentiated Lomax Exponential, Lomax Weibull, Lomax Rayleigh Lomax exponential, Weibull, Rayleigh, exponential and log-logistic distributions as special cases. A comprehensive investigation of the properties of this generalized distribution including series expansion of probability density function and cumulative distribution function, hazard and reverse hazard functions, quantile function, moments, conditional moments, mean deviations, Bonferroni and Lorenz curves, R'enyi entropy and distribution of order statistics are presented. Parameters of the model are estimated using maximum likelihood estimation technique and real data sets are used to illustrate the usefulness and applicability of the new generalized distribution compared with other distributions.
\end{abstract}

KEYWORDS :Burr XII distribution, Exponentiated Distribution, Maximum Likelihood Estimation, Weibull Distribution

\section{Introduction}

Burr XII distribution is a well known parametric model that has found wide application in different fields such as reliability, income and biological sciences. The hazard function of Weibull distribution is monotonically increasing, decreasing or constant which is disadvantageous because practical situations exhibit bath-tub shaped hazard function. Therefore, we propose a model that is flexible in accommodating various hazard functions which are likely to be encountered in practical situations. Burr XII distribution is a continuous distribution proposed by Irving W. Burr [4] as a two parameter 
distribution. Tadikamalla [30] added a scale parameter. The use of Burr XII distribution in accelerated life testing models was presented by Lewis [14]. Also, Hamid [8] studied Burr XII distribution under progressive Type-II censoring. Burr XII distribution is one of the most important and most widely used distribution for lifetime as well as the wealth data analysis and modeling. The usefulness and applicability of Burr XII distribution were studied by various authors including Hatke [9], Burr and Cislak [5], Rodrigues [28], Abd-Elfattah et al. [2] and Tadikamalla [30]. Burr XII distribution has been used in many applications such as actuarial science, quantal bio- assay, economics, forestry, toxicology, life testing and reliability. Mdlongwa et al. [16] eluded to the fact that this distribution covers shapes that is characteristic of a large number of distributions. The versatility and flexibility of the Burr XII distribution makes it quite attractive as a tentative model for data whose underlying distribution is unknown. Weibull distribution may be an initial choice when modeling monotone failure rates because of its negatively and positively skewed density shapes, however, it does not furnish a reasonable parametric fit for modeling practical situations with non-monotonic failure rates that are very useful in reliability and survival analysis.

According to the literature, there are several useful and important ways of developing new probability distributions from classic ones to relative new distributions. Nelson [20] wrote and discussed that distributions with bathtub-shaped failure rate are sufficiently complex and therefore difficult to model. The distribution proposed by Hjorth [11] is such an example. A review of these distributions was presented by Rajarshi and Rajarshi [27]. Haupt and Schabe [10] developed a new lifetime model with bathtub-shaped failure rates. It has been shown in the literature that exponentiated distributions are flexible enough to accommodate in many cases both monotone as well as bath-tub hazard rates. As a result many lifetime models extended Weibull and Burr XII distributions. Mudholkar and Hutson [17] applied the exponentiated Weibull distribution to model survival data and discussed that the generalized Weibull distribution has monotonically increasing, decreasing, bathtub and unimodal hazard function. Properties and the application of the generalized Weibull distribution was proposed by Mudholkar et al. [19]. For a review of these models the reader can refer to Mudholkar and Srivastava [18] and Pham and Lai [25], where the authors summarized some generalizations of Weibull distribution in their papers. Additional results on generalized distributions are given by Oluyede and Yang [23] on the beta generalized Lindley distribution, Oluyede et al. [22] on the gamma-Dagum distribution and the general family of univariate distributions generated from Weibull distribution that was introduced by Gurvich et al. [7]. Paranaiba et al. [24] proposed the beta Burr XII in order to add flexibility to the Burr XII distribution.

The primary motivation for the development of the exponentiated Burr XII Weibull distribution is the modeling of lifetime data and other data types with a diverse model that takes into consideration not only shape and scale, but also skewness, kurtosis and tail variation. Also, motivated by various applications of log-logistic,Weibull and beta distributions in several areas including reliability, exponential tilting (weighting) in finance and actuarial sciences, as well as economics, where Burr XII 
distribution plays an important role in income, we construct and develop the statistical properties of this new class of generalized distribution called the exponentiated Burr XII Weibull distribution and apply it to real lifetime data in order to demonstrate the usefulness of the proposed distribution.

In this paper, the results are organized in the following manner. The exponentiated Burr XII Weibull distribution, expansion of the density function, sub-models, quantile function, hazard and reverse hazard functions are given in Section 2. In Section 3, moments and conditional moments are presented. Mean deviations, Lorenz and Bonferroni curves are given in Section 4. Section 5 contains results on R'enyi entropy, density of the order statistics and L-moments. Maximum likelihood estimates of the model parameters are given in Section 6. A Monte Carlo simulation study to examine the bias and mean square error of the maximum likelihood estimates are presented in Section 7. Section 8 contains applications of the new model to real data sets. A short conclusion is given in Section 9.

\section{The Model: Exponentiated Burr XII Weibull Distribution}

In general, the generalized or exponentiated Burr XII Weibull (GBW or EBW) distribution is $G_{E B W}(x)=\left[F_{B W}(x)\right]^{\delta}$, where

$$
F_{B W}(x)=1-\left(1+x^{c}\right)^{-k} e^{-\alpha x^{\beta}}
$$

is a baseline Burr XII-Weibull cdf and $\delta>0$. The corresponding pdf is given by

$$
g_{E B W}(x)=\delta\left[F_{B W}(x)\right]^{\delta-1} f_{B W}(x) .
$$

For large values of $\mathrm{x}$, and for $\delta>1(<1)$, the multiplicative factor $\delta\left[F_{B W}(x)\right]^{\delta-1}>1(<1)$, respectively. The reverse statement holds for smaller values of $x$. Consequently, this implies that the ordinary moments of $g_{E B W}(x)$ are larger (smaller) than those of $F_{B W}(x)$ when $\delta>1(<1)$.

A generalization of the Burr XII and Weibull distributions where competing risk and exponentiation techniques are used to obtain the new exponentiated Burr XII Weibull (EBW) distribution with cumulative distribution function (cdf) that can be written as

$$
G_{E B W}(x ; c, k, \alpha, \beta, \delta)=\left(1-\left(1+x^{c}\right)^{-k} e^{-\alpha x^{\beta}}\right)^{\delta}, c, k, \alpha, \beta, \delta>0 .
$$

The corresponding probability density function (pdf) is given by

$$
\begin{gathered}
g_{E B W}(x ; c, k, \alpha, \beta, \delta) \\
=\delta\left(1-\left(1+x^{c}\right)^{-k} e^{-\alpha x^{\beta}}\right)^{\delta-1}\left(1+x^{c}\right)^{-k-1} e^{-\alpha x^{\beta}} \\
\times\left(k c x^{c-1}+\left(1+x^{c}\right)-\alpha \beta x^{\beta-1}\right)
\end{gathered}
$$

for $x \geq 0, c, k, \alpha, \beta, \delta>0$. We note that the parameter $\alpha$ control the scale of the distribution and the parameters $c, k, \beta$ and $\delta$ controls the shape of the distribution, respectively.

Plots of the EBW pdf for selected values of the model parameters are given in Figure 1. The plots show that the EBW distribution can be L-shaped or decreasing and right skewed among many potential shapes. The reliability or survival function of the EBW distribution is given by 


$$
\begin{gathered}
\bar{G}_{E B W}(x ; c, k, \alpha, \beta, \delta) \\
=\left[1-\left(1+x^{c}\right)^{-k} e^{-\alpha x^{\beta}}\right]^{\delta}, c, k, \alpha, \beta \text {, and } \delta>0 .
\end{gathered}
$$

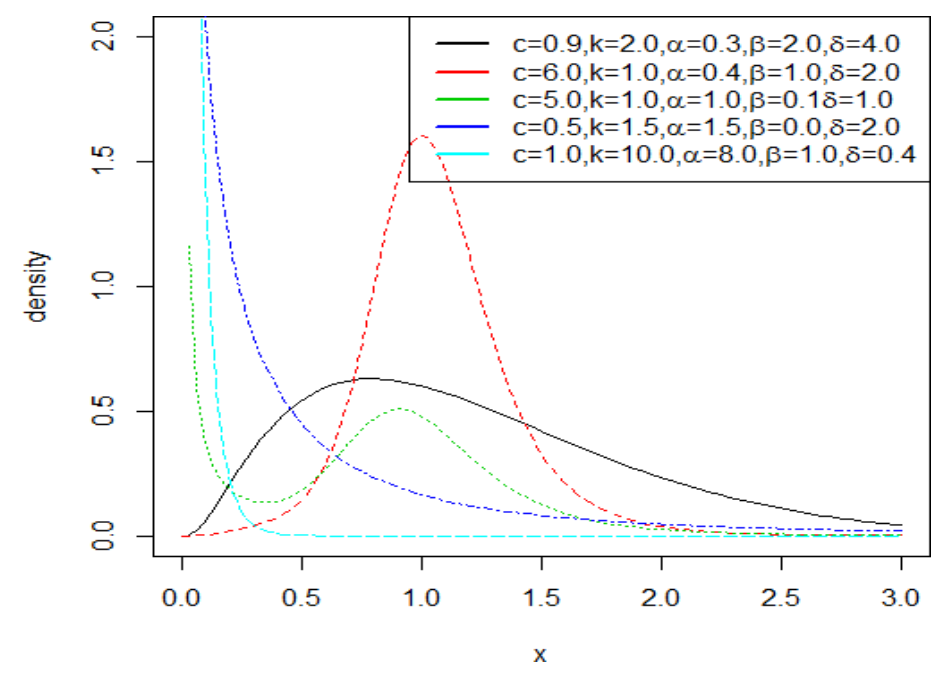

Figure 1. Plots of the pdf of the EBW distribution for selected values of the model parameters

\subsection{Expansion of Density Function}

In this subsection, we present a series expansion of the EBW density function. Using the generalization of binomial theorem : If $\mathrm{b}$ is a positive real non-integer and $|z|<1$, then

$$
(1-z)^{b-1}=\sum_{j=0}^{\infty} \frac{(-1)^{j}}{\Gamma(b-j) j !} z^{j}
$$

We have the expression

$$
\left(1-\left(1+x^{c}\right)^{-k} e^{-\alpha x^{\beta}}\right)^{\delta-1}=\sum_{j=0}^{\infty} \frac{(-1)^{j} \Gamma(\delta)}{\mathrm{j} ! \Gamma(\delta-j) j}\left(\left(1+x^{c}\right)^{-k} e^{-\alpha x^{\beta}}\right)^{j},
$$

so we can write the EBW pdf as

$$
\begin{aligned}
g_{E B W}(x)= & \sum_{j=0}^{\infty} \frac{(-1)^{j} \Gamma(\delta+1)}{j ! \Gamma(\delta-j) j}\left(1+x^{c}\right)^{-k j} e^{-\alpha j x^{\beta}} \\
& \times\left(1+x^{c}\right)^{-k-1} e^{-\alpha x^{\beta}}\left[k c x^{c-1}+\left(1+x^{c}\right) \alpha \beta x^{\beta-1}\right] \\
= & \sum_{j=0}^{\infty} \frac{(-1)^{j} \Gamma(\delta+1)}{(j+1) j ! \Gamma(\delta-j)} \times(j+1)\left(\bar{G}_{B W}(X)\right)^{j+1-1} g_{B W}(X) \\
= & \sum_{j=0}^{\infty} w(\delta, j) \times(j+1)\left(\bar{G}_{B W}(X)\right)^{j+1-1} g_{B W}(X),
\end{aligned}
$$


where

$$
\mathrm{w}(\delta, \mathrm{j})=\frac{(-1)^{j} \Gamma(\delta+1)}{(j+1) j ! \Gamma(\delta-j)},
$$

and $\bar{G}_{B W}(x)$, and $g_{B W}(x)$ are the reliability function and pdf of the BW distribution with the parameters $\mathrm{c}, \mathrm{k}, \alpha$ and $\beta$, respectively.

\subsection{Hazard and Reverse Hazard Functions}

The hazard and reverse hazard functions of the EBW distribution are respectively given by

$$
\begin{aligned}
h_{G}(x) & =\frac{g_{E B W}(x)}{\bar{G}_{\mathrm{E} B W}(x)} \\
& =\frac{\delta\left(1-\left(1+x^{c}\right)^{-k} e^{-\alpha x^{\beta}}\right)^{\delta-1}\left(1+x^{c}\right)^{-k-1} e^{-\alpha x^{\beta}}\left(k c x^{c-1}+\left(1+x^{c}\right) \alpha \beta x^{\beta-1}\right)}{1-\left[1-\left(1+x^{c}\right)^{-k} e^{-\alpha x^{\beta}}\right]^{\delta}}
\end{aligned}
$$

and

$T_{G}(x)=\frac{g_{E B W}(x)}{\mathrm{G}_{\mathrm{EBW}}(x)}$

$=\delta\left[1-\left(1+x^{c}\right)^{-k} e^{-\alpha x^{\beta}}\right]^{-1}\left(1+x^{c}\right)^{-k-1} e^{-\alpha x^{\beta}}\left[k c x^{c-1}+\left(1+x^{c}\right) \alpha \beta x^{\beta-1}\right]$.

Plots of the hazard function for selected values of the EBW parameters are also presented in Figure

2. The graphs of the hazard function show that EBW distribution

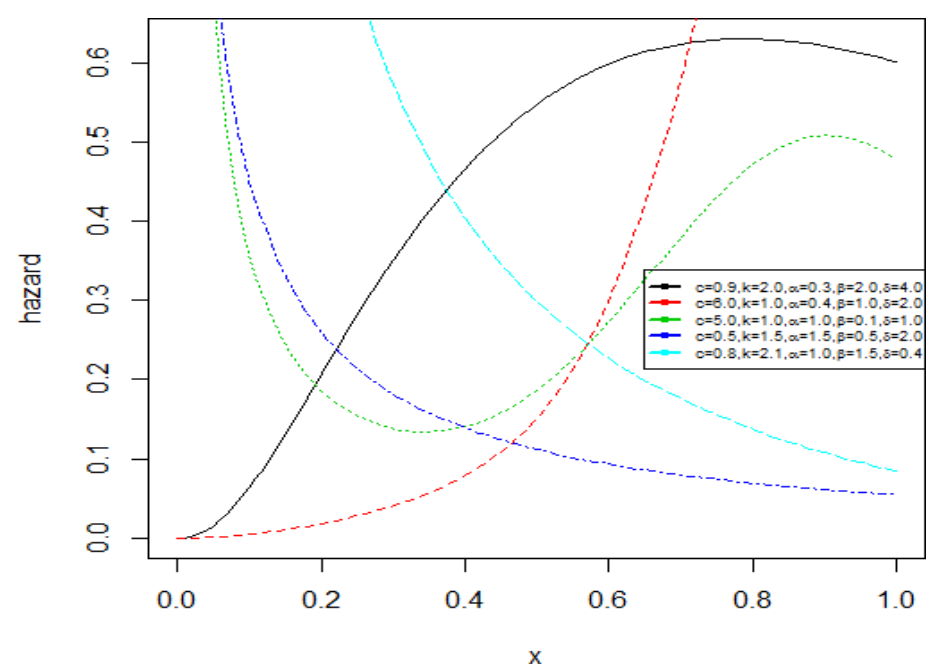

Figure 2. Graphs of the hazard of the EBW distribution for selected values of the model parameters

have different shapes including monotonically increasing, monotonically decreasing and bathtub followed by upside down bathtub shapes. These shapes of the hazard function show that EBW distribution can accommodate both monotonic and non-monotonic hazard behaviors which are more likely to be encountered when dealing with survival and lifetime data. 


\subsection{Some Sub-models}

There are several new and well known distributions that can be obtained from the EBW distribution. The sub-models include the following distributions :

- When $\beta=1$, we obtain Exponentiated Burr XII Exponential (EBE) distribution.

The EBE cdf is defined by

$$
G_{E B W}(x ; c, k, \alpha, \delta)=\left(1-\left(1+x^{c}\right)^{-k}-e^{-\alpha x}\right)^{\delta}, c, k, \alpha, \delta>0 .
$$

- When $\beta=2$, we obtain Exponentiated Burr XII Rayleigh (EBR) distribution.

The EBR cdf is given by

$$
G_{E B W}(x ; c, k, \alpha, \delta)=\left(1-\left(1+x^{c}\right)^{-k}-e^{-\alpha x^{2}}\right)^{\delta}, c, k, \alpha, \delta>0 .
$$

- When $\alpha \rightarrow 0^{+}$and $\beta=1$, then we have the Exponentiated Burr XII (EB) distribution. The EB cdf is defined by

$$
G_{E B}(x ; c, k, \delta)=\left(1-\left(1+x^{c}\right)^{-k}\right)^{\delta}, c, k, \delta>0 .
$$

- When $\mathrm{k}=\beta=1$, and $\alpha \rightarrow 0^{+}$, we obtain Exponentiated Log-logistic (ELLoG) distribution. The ELLoG cdf is defined by

$$
G_{E L L o G}(x ; c, \delta)=\left(1-\left(1+x^{c}\right)^{-1}\right)^{\delta}, c, \delta>0 .
$$

- When $\mathrm{k} \rightarrow 0^{+}$, we obtain Exponentiated Weibull (EW) distribution (Mudholkar and Srivastava [18]). The EW cdf is defined by

$$
G_{E W}(x ; \delta, \alpha, \beta)=\left(1-e^{-\alpha x^{\beta}}\right)^{\delta}, \quad \delta, \alpha, \beta>0 .
$$

- When $\mathrm{k} \rightarrow 0^{+}$, and $\beta=2$, we obtain Exponentiated Rayleigh (ER) distribution (Mudholkar and Srivastava [18]). The cdf of ER distribution is given by

$$
G_{E R}(x ; \delta, \alpha)=\left(1-e^{-\alpha x^{2}}\right)^{\delta}, \quad \delta, \alpha>0 .
$$

- When $\mathrm{k} \rightarrow 0^{+}$, and $\beta=1$, then we have the Exponentiated Exponential (EE) distribution (Gupta et al. [6]). The cdf of EE distribution is given by

$$
G_{E E}(x ; \alpha, \delta)=\left(1-e^{-\alpha x}\right)^{\delta}, \quad \delta, \alpha>0 .
$$

- When $\mathrm{c}=1$, we have Exponentiated Lomax-Weibull (ELW) distribution. The cdf of the ELW distribution is given by

$$
G_{E L W}(x ; k, \alpha, \beta, \delta)=\left(1-(1+x)^{-k} e^{-\alpha x^{\beta}}\right)^{\delta}, \quad k, \alpha, \beta, \delta>0 .
$$

- When $c=1$ and $\beta=2$, we obtain Exponentiated Lomax-Rayleigh (ELR) distribution. The cdf of the ELR distribution is defined by

$$
G_{E L R}(x ; k, \alpha, \delta)=\left(1-(1+x)^{-k} e^{-\alpha x^{2}}\right)^{\delta}, \quad k, \alpha, \delta>0 .
$$

- When $\mathrm{c}=\beta=1$, we obtain Exponentiated Lomax-Exponential (ELE) distribution.

The cdf of the ELE distribution is given by

$$
G_{E L E}(x ; k, \alpha, \delta)=\left(1-(1+x)^{-k} e^{-\alpha x}\right)^{\delta}, \quad k, \alpha, \delta>0 .
$$

- When $\mathrm{c}=1$, and $\alpha \rightarrow 0^{+}$, we have Exponentiated Lomax (EL) distribution (Abdul-Moniem and Abdel-Hameed [1]). The cdf of the EL distribution is given by

$$
G_{E L}(x ; k, \delta)=\left(1-(1+x)^{-k}\right)^{\delta}, \quad k, \delta>0 .
$$

- When $\delta=1$, we obtain Burr XII Weibull (BW) distribution and the cdf is given by 


$$
G_{B W}(x ; c, k, \alpha, \beta)=1-\left(1+x^{c}\right)^{-k} e^{-\alpha x^{\beta}}, \quad c, k, \alpha, \beta>0 .
$$

- When $\delta=\beta=1$, then we have Burr XII exponential (BE) distribution and the cdf is given by

$$
G_{B E}(x ; c, k, \alpha)=1-\left(1+x^{c}\right)^{-k} e^{-\alpha x}, \quad c, k, \alpha>0 .
$$

- When $\delta=1$ and $\beta=2$, we obtain Burr XII Rayleigh (BR) distribution and the cdf is defined by

$$
G_{B R}(x ; c, k, \alpha)=1-\left(1+x^{c}\right)^{-k} e^{-\alpha x^{2}}, \quad c, k, \alpha>0 .
$$

- When $\delta=\mathrm{k}=1$, we obtain Log-logistic Weibull (LLoGW) distribution and the cdf is defined by

$$
G_{L L o G W}(x ; c, \alpha, \beta)=1-\left(1+x^{c}\right)^{-1} e^{-\alpha x^{\beta}}, \quad c, \alpha, \beta>0 .
$$

- When $\delta=\beta=\mathrm{k}=1$, we have Log-logistic exponential (LLoGE) distribution and the cdf is defined by

$$
G_{L L O G E}(x ; c, \alpha)=1-\left(1+x^{c}\right)^{-1} e^{-\alpha x}, \quad c, \alpha>0 .
$$

- When $\delta=\mathrm{k}=1$ and $\beta=2$, we have Log-logistic Rayleigh (LLoGR) distribution and the cdf is defined by

$$
G_{L L o G R}(x ; c, \alpha)=1-\left(1+x^{c}\right)^{-1} e^{-\alpha x^{2}}, \quad c, \alpha>0 .
$$

- When $\delta=\mathrm{c}=1$, we obtain Lomax-Weibull (LW) distribution and the cdf is defined by

$$
G_{L W}(x ; k, \alpha, \beta)=1-(1+x)^{-k} e^{-\alpha x^{\beta}}, \quad k, \alpha, \beta>0 .
$$

- When $\delta=\beta=\mathrm{c}=1$, we obtain Lomax-Exponential distribution (LE) distribution and the cdf is given by

$$
G_{L E}(x ; k, \alpha)=1-(1+x)^{-k} e^{-\alpha x}, \quad k, \alpha>0 .
$$

- When $\delta=1$, and $\mathrm{k} \rightarrow 0^{+}$, we obtain Weibull (W) distribution and the cdf is given by

$$
G_{W}(x ; \alpha, \beta)=1-e^{-\alpha x^{\beta}}, \quad \alpha, \beta>0 .
$$

- When $\delta=\beta=1$, and $\mathrm{k} \rightarrow 0^{+}$, we obtain Exponential (E) distribution and the cdf is given by

$$
G_{E}(x ; \alpha)=1-e^{-\alpha x}, \quad \alpha>0 .
$$

- When $\delta=1, \beta=2$, and $\mathrm{k} \rightarrow 0^{+}$, we obtain Rayleigh distribution and the cdf is given by

$$
G_{R}(x ; \alpha)=1-e^{-\alpha x^{2}}, \quad \alpha>0 .
$$

- When $\alpha \rightarrow 0^{+}$, we obtain Burr XII (B) distribution and the cdf is given by

$$
G_{B}(x ; c, k)=1-\left(1+x^{c}\right)^{-k}, \quad c, k>0 .
$$

- When $\alpha \rightarrow 0^{+}, \delta=1$, and $\mathrm{k}=1$, we have Log-logistic (LLoG) distribution and the cdf is given by

$$
G_{L L O G}(x ; c)=1-\left(1+x^{c}\right)^{-1}, \quad c>0 .
$$

- When $\alpha \rightarrow 0^{+}, \delta=1$, and $\mathrm{c}=1$, we have the Lomax (L) distribution and the cdf is given by

$$
G_{L}(x ; k)=1-(1+x)^{-k}, \quad k>0 .
$$




\subsection{Quantile Function}

To obtain the quantile function of the EBW distribution, we invert the following

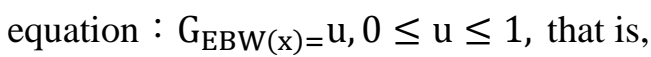

$$
\left[1-\left(1+x^{c}\right)^{-k} e^{-\alpha x^{\beta}}\right]^{\delta}=u,
$$

so that,

$$
\left(1+x^{c}\right)^{-k} e^{-\alpha x^{\beta}}=1-u^{\frac{1}{\delta}}
$$

and

$$
\alpha x^{\beta}+k \log \left(1+x^{c}\right)+\log \left(1-u^{\frac{1}{\delta}}\right)=0 .
$$

We can obtain the quantile function of the EBW distribution by solving the equation (32) using numerical methods. Equation (32) can be used to generate random number. The quantiles for selected values of the EBW distribution parameters are listed in Table 1.

Table 1. EBW quantile for selected values

\begin{tabular}{cccccc}
\hline \multicolumn{5}{c}{$(c, k, \delta, \alpha, \beta)$} \\
\hline $\mathrm{u}$ & $(0.8,1.2,1.5,0.9,1.0)$ & $(1.2,1.8,1.2,2.0,3.0)$ & $(5.5,0.6,0.3,0.5,1.0)$ & $(0.3,1.82,3.0,6.0,2.0)$ & $(1.8,1.2,0.8,0.6,3.0)$ \\
\hline 0.1 & 0.0539 & 0.1336 & 0.0009 & 0.0464 & 0.1814 \\
0.2 & 0.1062 & 0.2265 & 0.0094 & 0.1264 & 0.3000 \\
0.3 & 0.1650 & 0.3112 & 0.0365 & 0.1974 & 0.4020 \\
0.4 & 0.2342 & 0.3921 & 0.0966 & 0.2596 & 0.5040 \\
0.5 & 0.3186 & 0.4720 & 0.2100 & 0.3171 & 0.6100 \\
0.6 & 0.4262 & 0.5540 & 0.3940 & 0.3744 & 0.7191 \\
0.7 & 0.5719 & 0.6412 & 0.6305 & 0.4360 & 0.8440 \\
0.8 & 0.7905 & 0.7430 & 0.8584 & 0.5082 & 0.998 \\
0.9 & 1.1978 & 0.8797 & 1.1304 & 0.6103 & 1.2030 \\
\hline
\end{tabular}

\section{Moments}

In statistics, moments are used to characterize various features of a distribution, for example skewness and kurtosis. The $t^{\text {th }}$ non central moment of the EBW distribution is given by

$$
\begin{aligned}
E\left(X^{t}\right) & =\int_{0}^{\infty} x^{t} g_{E B W}(x) d x \\
& =\int_{0}^{\infty} x^{t} \delta\left(1-\left(1+x^{c}\right)^{-k} e^{-\alpha x^{\beta}}\right)^{\delta-1}\left(1+x^{c}\right)^{-k-1} e^{-\alpha x^{\beta}} \\
& \times\left(k c x^{c-1}+\left(1+x^{c}\right) \alpha \beta x^{\beta-1}\right) d x .
\end{aligned}
$$


Note that from the generalized binomial theorem : If $\mathrm{b}$ is a positive real non-integer and $|z|<1$, then

$$
(1-z)^{b-1}=\sum_{j=0}^{\infty} \frac{(-1)^{j} \Gamma(b)}{\Gamma(b-j) j !}
$$

we have the expression

$$
\left(1-\left(1+x^{c}\right)^{-k} e^{-\alpha x^{\beta}}\right)^{\delta-1}=\sum_{j=0}^{\infty} \frac{(-1)^{j} \Gamma(\delta)}{j ! \Gamma(\delta-j)}\left(\left(1+x^{c}\right)^{-k} e^{-\alpha x^{\beta}}\right)^{j} .
$$

Thus, the $\mathrm{t}^{\text {th }}$ moment of the EBW distribution can be obtained as follows :

$$
\begin{aligned}
E\left(X^{t}\right) & =\int_{0}^{\infty} x^{t} g_{E B W}(x) d x \\
& =\sum_{j=0}^{\infty} \frac{(-1)^{j} \Gamma(\delta+1)}{j ! \Gamma(\delta-j)} \int_{0}^{\infty} x^{t}\left(1+x^{c}\right)^{-k j-k-1} e^{-\alpha(j+1) x^{\beta}} \\
& \times\left[k c x^{c-1}+\left(1+x^{c}\right) \alpha \beta x^{\beta-1}\right] d x .
\end{aligned}
$$

Now, we apply the expansion $e^{x}=\sum_{p=0}^{\infty} \frac{x^{p}}{p !}$ To obtain

$$
\begin{aligned}
& E\left(X^{t}\right)=\sum_{j=0}^{\infty} \sum_{p=0}^{\infty} \frac{(-1)^{j+p}[\alpha(j+1)]^{p} \Gamma(\delta+1)}{p ! j ! \Gamma(\delta-j)} \\
& {\left[k c \int_{0}^{\infty} x^{t+p \beta+c-1\left(1+x^{c}\right)^{-k j-k-1}} d x+\alpha \beta \int_{0}^{\infty} x^{t+p \beta+\beta-1\left(1+x^{c}\right)^{-k j-k}} d x\right] .} \\
& \text { Let } y=\left(1+x^{c}\right)^{-1} \text {, then } x=\left(\frac{1-y}{y}\right)^{\frac{1}{c}} \text { and } \\
& d x=-\frac{y^{-2}(1-y)^{\frac{1}{c}-1} y^{1-\frac{1}{c}}}{c} d y \text {, so that } \\
& \mathrm{E}\left(X^{t}\right)=\sum_{j=0}^{\infty} \sum_{p=0}^{\infty} \frac{(-1)^{j+p}[\alpha(j+1)]^{p} \Gamma(\delta+1)}{p ! j ! \Gamma(\delta-j)} \\
& \times\left[k c \int_{0}^{1}\left[\left(\frac{1-y}{y}\right)^{\frac{1}{c}}\right]^{t+P \beta+c-1} y^{k j+k+1} \frac{y^{-2}(1-y)^{\frac{1}{c}-1} y^{1-\frac{1}{c}}}{c} d y\right. \\
& \left.\left.+\alpha \beta \int_{0}^{1}\left(\frac{1-y}{y}\right)^{\frac{1}{c}}\right]^{t+p \beta+\beta-1} y^{k j+k} \frac{y^{-2}(1-y)^{\frac{1}{c}-1} y^{1-\frac{1}{c}}}{c} d y\right] \\
& =\sum_{j=0}^{\infty} \sum_{p=0}^{\infty} \frac{(-1)^{j+p}[\alpha(j+1)]^{p} \Gamma(\delta+1)}{p ! j ! \Gamma(\delta-j)} \\
& {\left[\mathrm{k} \int_{0}^{1} y^{k j+k-\frac{t}{c}-\frac{p \beta}{c}-1}(1-y)^{\frac{t+p \beta+c}{c}-1} d y+\frac{\alpha \beta}{c} \int_{0}^{1} y^{k j+k-\frac{t}{c}-\frac{p \beta}{c}-\frac{\beta}{c}-1}(1-y)^{\frac{t+p \beta+\beta}{c}-1} d y\right]}
\end{aligned}
$$




$$
\begin{aligned}
& =\sum_{j=0}^{\infty} \sum_{p=0}^{\infty} \frac{(-1)^{j+p}[\alpha(j+1)]^{p} \Gamma(\delta+1)}{p ! j ! \Gamma(\delta-j)} k B\left(k j+k-\frac{t}{c}-\frac{p \beta}{c}, \frac{t+p \beta+c}{c}\right) \\
& +\frac{\alpha \beta}{c} B\left(k j+k-\frac{t}{c}-\frac{p \beta}{c}-\frac{\beta}{c}, \frac{t+p \beta+\beta}{c}\right),
\end{aligned}
$$

where $\mathrm{B}(\mathrm{a}, \mathrm{b})=\int_{0}^{1} \mathrm{z}^{\mathrm{a}-1}(1-\mathrm{z})^{\mathrm{b}-1} \mathrm{dz}$ denote the complete beta function.

The first six moments $\left(\mu_{1}^{\prime}, \mu_{2}^{\prime}, \mu_{3}^{\prime}, \mu_{4}^{\prime}, \mu_{5}^{\prime}, \mu_{6}^{\prime}\right)$, standard deviation (SD), coefficient of variation (CV), coefficient of skewness (CS) and coefficient of kurtosis (CK) for different selected values of the EBW distribution parameters are listed in Table 2.

Table 2. EBW Moments for selected values

\begin{tabular}{cccccc}
\hline \multicolumn{5}{c}{$(\mathrm{c}, \mathrm{k}, \delta, \alpha, \beta)$} & \\
\hline Moments & $(1.0,3.0,2.0,3.0,1.0)$ & $(1.0,3.0,1.0,1.5,2.0)$ & $(1.0,3.0,0.9,1.5,1.0)$ & $(0.9,2,0.8,0.5,2.0)$ & $(1.8,1.2,0.8,0.6,3.0)$ \\
\hline$\mu_{1}^{\prime}$ & 0.2721 & 0.3068 & 0.2367 & 0.4303 & 0.2173 \\
$\mu_{2}^{\prime}$ & 0.1220 & 0.1708 & 0.1338 & 0.4162 & 0.1256 \\
$\mu_{3}^{\prime}$ & 0.0794 & 0.1301 & 0.1284 & 0.5738 & 0.1149 \\
$\mu_{4}^{\prime}$ & 0.0699 & 0.1218 & 0.1820 & 0.9791 & 0.1435 \\
$\mu_{5}^{\prime}$ & 0.0791 & 0.1325 & 0.3504 & 1.9405 & 0.2270 \\
$\mu_{6}^{\prime}$ & 0.1107 & 0.1622 & 0.8668 & 4.3137 & 0.4345 \\
$\mathrm{SD}$ & 0.2188 & 0.2769 & 0.2786 & 0.4807 & 0.2800 \\
$\mathrm{CV}$ & 0.8040 & 0.9023 & 1.1769 & 1.1170 & 1.2887 \\
$\mathrm{CS}$ & 1.9297 & 1.4450 & 2.7730 & 1.7638 & 2.4412 \\
$\mathrm{CK}$ & 9.2540 & 5.4392 & 15.9184 & 6.5761 & 11.8037 \\
\hline
\end{tabular}



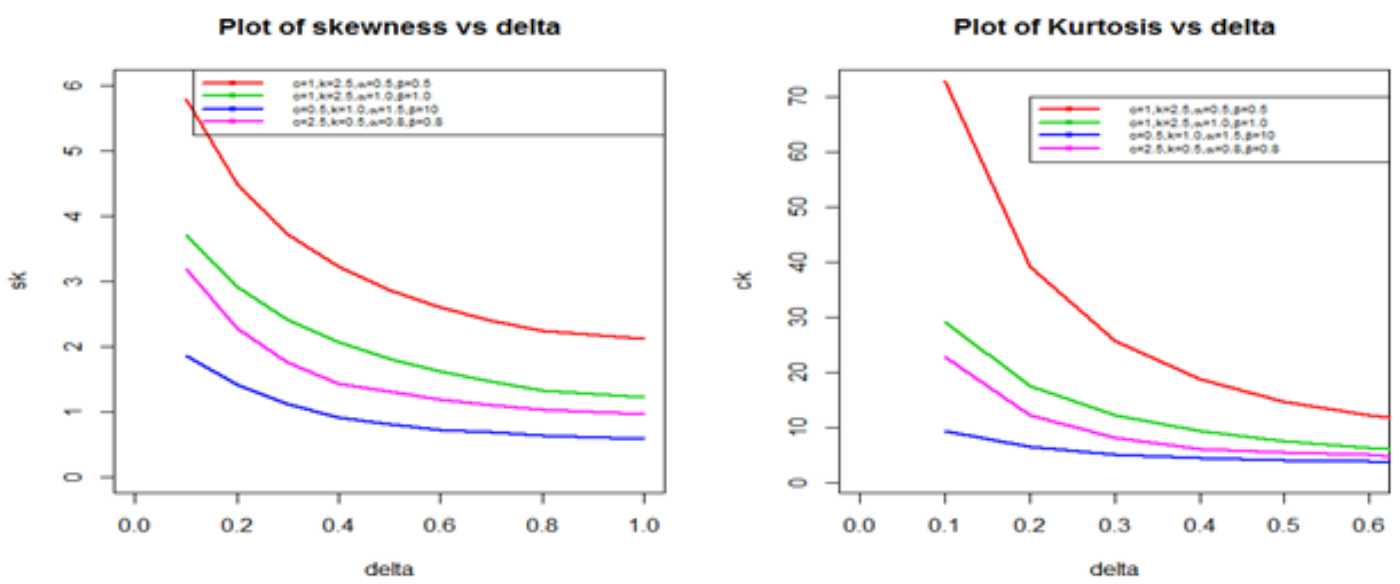

Figure3. Plot of Skewness and Kurtosis for selected values EBW distribution parameters
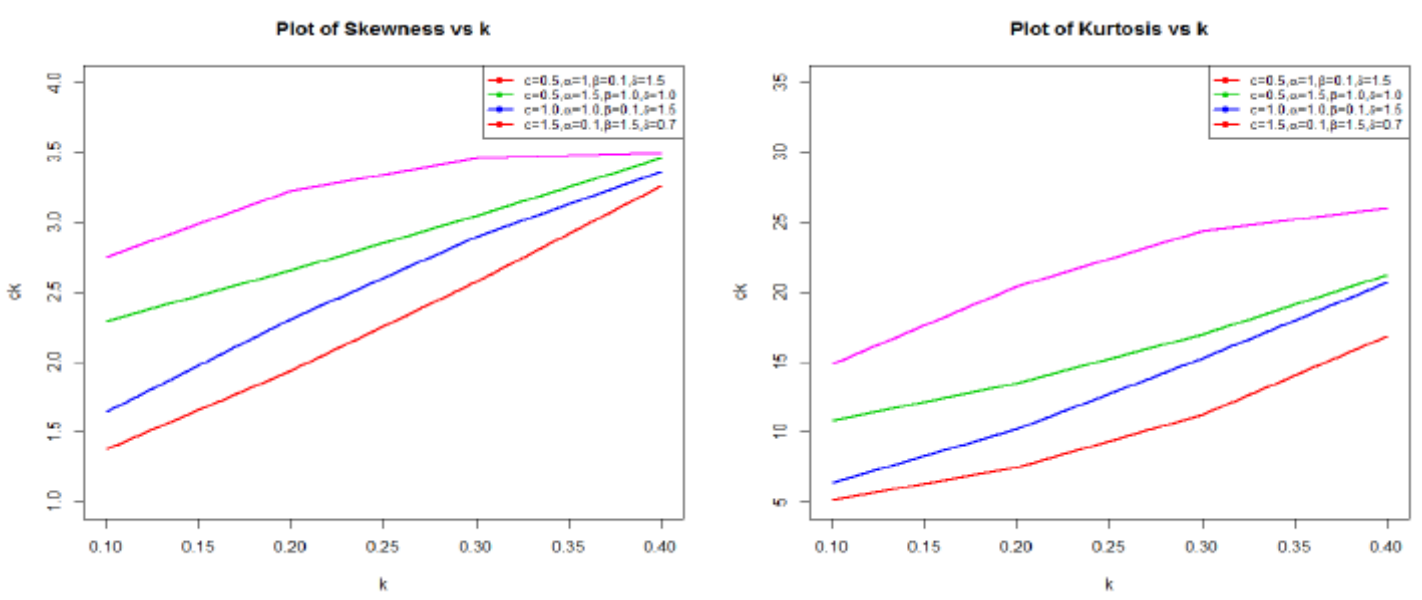

Figure4. Plot of Skewness and Kurtosis for selected values EBW distribution parameters
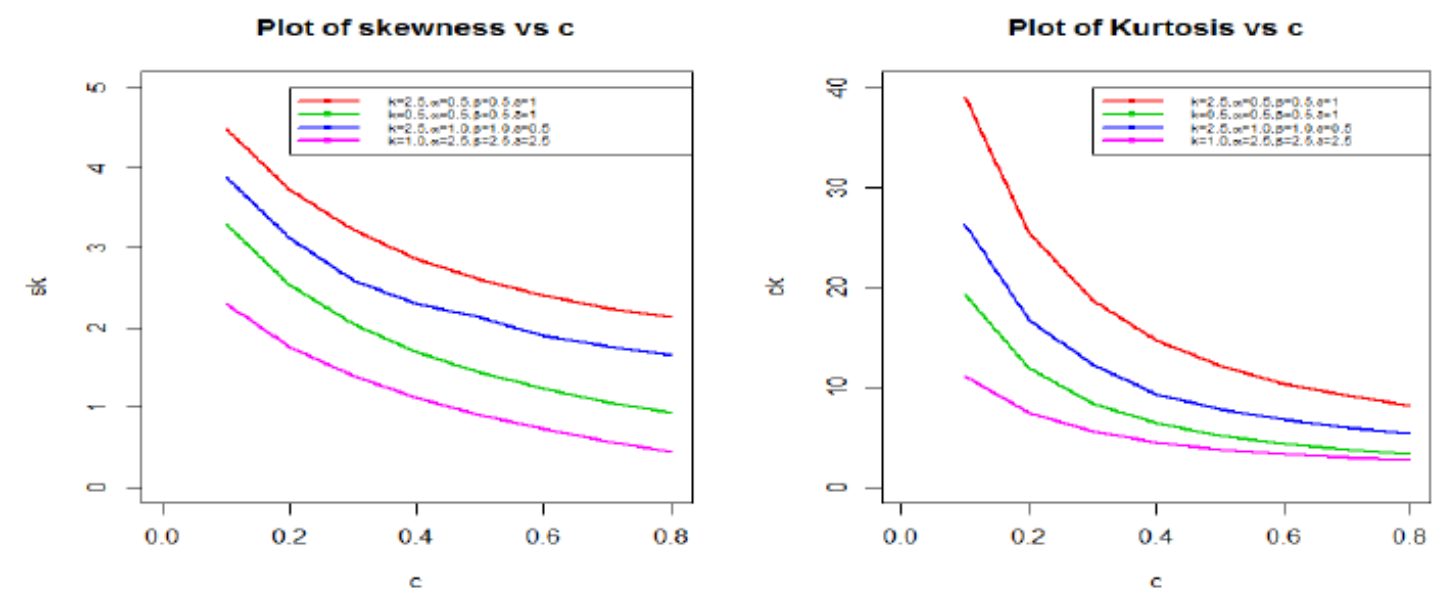

Figure5. Plot of Skewness and Kurtosis for selected values EBW distribution parameters 

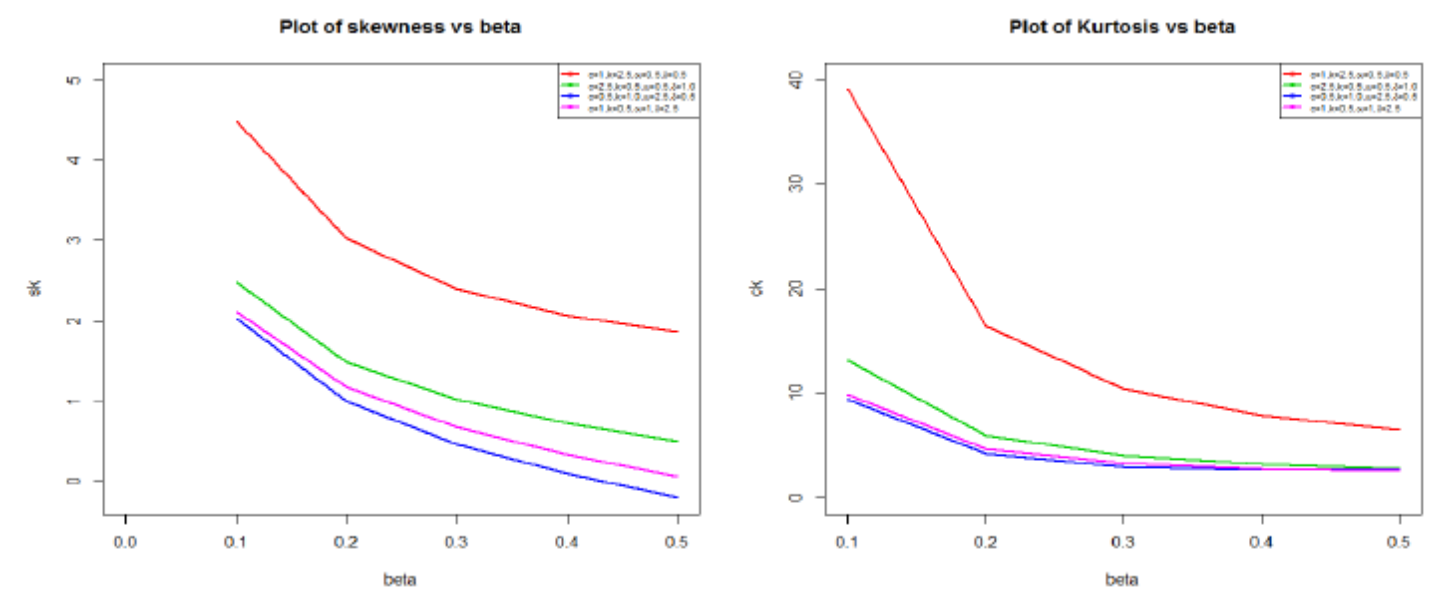

Figure6. Plot of Skewness and Kurtosis for selected values EBW distribution parameters

Graphs of skewness and kurtosis of the EBW distribution as a function of different model shape parameters are presented. The plots show the dependence of the kurtosis and skewness measures of the shape parameter $: \delta, \mathrm{k}, \mathrm{c}$ and $\beta$, respectively. Plot of the skewness and kurtosis decreases as $\mathrm{c}, \beta$ and $\delta$ increase while the graph of the skewness and kurtosis increase as parameter $\mathrm{k}$ increases.

\subsection{Conditional Moments}

The $\mathrm{t}^{\text {th }}$ conditional moment of the EBW distribution is given by

$$
\begin{aligned}
\mathrm{E}\left(X^{t} \mid \mathrm{X}>\mathrm{r}\right) & =\frac{1}{\bar{G}_{E B W(r)}} \int_{r}^{\infty} x^{t} g_{E B W}(x) d x \\
& =\frac{1}{\bar{G}_{E B W(r)}} \int_{r}^{\infty} x^{t} \delta\left(1-\left(1+x^{c}\right)^{-k} e^{-\alpha x^{\beta}}\right)^{\delta-1}\left(1+x^{c}\right)^{-k-1} e^{-\alpha x^{\beta}} \\
& \times\left(k c x^{c-1}+\left(1+x^{c}\right) \alpha \beta x^{\beta-1}\right) \mathrm{dx} \\
& =\frac{1}{\bar{G}_{E B W(r)}} \sum_{j=0}^{\infty} \sum_{p=0}^{\infty} \frac{(-1)^{j+P}[\alpha(j+1)]^{p} \Gamma(\delta+1)}{p ! j ! \Gamma(\delta-j)} \\
& \times \mathrm{k} B_{\left[1+r^{c}\right]^{-1}}\left(k j+k-\frac{t}{c}-\frac{p \beta}{c}, \frac{t+p \beta+c}{c}\right) \\
& +\frac{\alpha \beta}{c} B_{\left[1+r^{c}\right]^{-1}}\left(k j+k-\frac{t}{c}-\frac{p \beta}{c}-\frac{\beta}{c}, \frac{t+p \beta+\beta}{c}\right)
\end{aligned}
$$

Where $B_{y}(a, b)=\int_{0}^{y} z^{a-1}(1-z)^{b-1} d z$ is the incomplete beta function.

\section{Mean Deviations}

According to Stuart and Ord [29], the totality deviations from the mean $\mu$ and the median $M$ measures the amount spread in a population. The mean deviation about the mean measures the average 
absolute deviations of the observation from its mean and the mean deviation about the median measures the average absolute deviation of the observation from its median. The mean deviation about the mean and the mean deviation about the median are respectively given by

$$
\delta_{\mu}(x)=2 \mu G(\mu)-2 \mu+2 \int_{\mu}^{\infty} x g(x) d x \text { and } \delta_{M}(x)=-\mu+2 \int_{M}^{\infty} x g(x) d x .
$$

The mean deviation about the mean for the EBW distribution is given by :

$$
\delta_{\mu}(x)=2 \mu G_{E B W}(\mu)-2 u+2 T(\mu)
$$

Where

$$
\begin{aligned}
\mathrm{T}(\mu) & =\int_{\mu}^{\infty} x g_{E B W}(x) d x \\
& =\sum_{j=0}^{\infty} \sum_{p=0}^{\infty} \frac{(-1)^{j+p}[\alpha(j+1)]^{p} \Gamma(\delta+1)}{p ! j ! \Gamma(\delta-j)} \\
& \times k B_{\left[1+\mu^{e}\right]^{-1}}\left(k j+k-\frac{1}{c}-\frac{p \beta}{c}, \frac{1+p \beta+c}{c}\right) \\
& +\frac{\alpha \beta}{c} B_{\left[1+\mu^{e}\right]^{-1}}\left(k j+k-\frac{1}{c}-\frac{p \beta}{c}-\frac{\beta}{c}, \frac{1+p \beta+\beta}{c}\right) .
\end{aligned}
$$

The mean deviation about the median for the EBW distribution is given by

$$
\delta_{M}(x)=-\mu+2 T(M)
$$

where

$$
\begin{aligned}
T(M) & =\int_{M}^{\infty} x g_{E B W}(x) d x \\
& =\sum_{j=0}^{\infty} \sum_{p=0}^{\infty} \frac{(-1)^{j+p}[\alpha(j+1)]^{p} \Gamma(\delta+1)}{p ! j ! \Gamma(\delta-j)} \\
\times & k B_{\left[1+M^{e}\right]^{-1}}\left(k j+k-\frac{1}{c}-\frac{p \beta}{c}, \frac{1+p \beta+c}{c}\right) \\
+ & \frac{\alpha \beta}{c} B_{\left[1+M^{e}\right]^{-1}}\left(k j+k-\frac{1}{c}-\frac{p \beta}{c}-\frac{\beta}{c}, \frac{1+p \beta+\beta}{c}\right) .
\end{aligned}
$$

\subsection{Bonferroni and Lorenz Curves}

Bonferroni and Lorenz curves are presented in this section. These measures of in-equality are widely used in various fields such as survival analysis, demography and insurance. Bonferroni and Lorenz curves for the EBW distribution are given by

$$
B(p)=\frac{1}{p \mu} \int_{0}^{q} x g_{E B W}(x) d x=\frac{1}{p \mu}[\mu-T(q)]
$$




$$
\begin{aligned}
& =\frac{1}{p \mu}\left[\mu-\sum_{j=0}^{\infty} \sum_{p=0}^{\infty} \frac{(-1)^{j+p}[\alpha(j+1)]^{p} \Gamma(\delta+1)}{p ! j ! \Gamma(\delta-j)}\right. \\
& \times k B_{\left[1+q^{e}\right]^{-1}}\left(k j+k-\frac{1}{c}-\frac{p \beta}{c}, \frac{1+p \beta+c}{c}\right) \\
& \left.+\frac{\alpha \beta}{c} B_{\left[1+q^{e}\right]^{-1}}\left(k j+k-\frac{1}{c}-\frac{p \beta}{c}-\frac{\beta}{c}, \frac{1+p \beta+\beta}{c}\right)\right],
\end{aligned}
$$

and

$$
\begin{aligned}
L(p) & =\frac{1}{\mu} \int_{0}^{q} x g_{E B W}(x) d x=\frac{1}{\mu}[\mu-T(q)] \\
& =\frac{1}{\mu}\left[\mu-\sum_{j=0}^{\infty} \sum_{p=0}^{\infty} \frac{(-1)^{j+p}[\alpha(j+1)]^{p} \Gamma(\delta+1)}{p ! j ! \Gamma(\delta-j)}\right. \\
& \times k B_{\left[1+q^{e}\right]^{-1}}\left(k j+k-\frac{1}{c}-\frac{p \beta}{c}, \frac{1+p \beta+c}{c}\right) \\
& \left.+\frac{\alpha \beta}{c} B_{\left[1+q^{e}\right]^{-1}}\left(k j+k-\frac{1}{c}-\frac{p \beta}{c}-\frac{\beta}{c}, \frac{1+p \beta+\beta}{c}\right)\right],
\end{aligned}
$$

respectively.

\section{Rényi Entropy and Order Statistics}

The concept of entropy can be shown to be a good measure of uncertainty and plays a vital role in information theory. Rényi entropy and distribution of order statistics for the EBW distribution are presented in this section.

\subsection{Renyi Entropy}

Renyi entropy is given by

$$
\begin{aligned}
I_{R}(v)= & \frac{1}{1-v} \log \left(\int_{0}^{\infty}\left[g_{E B W}(x ; c, k, \alpha, \beta, \delta)\right]^{v} d x\right) \\
& , \boldsymbol{v} \neq \mathbf{1}, \boldsymbol{v}>\mathbf{0} .
\end{aligned}
$$

Note that,

$$
\begin{aligned}
{\left[g_{E B W}(x)\right]^{v} } & =\delta^{v}\left[1-\left(1+x^{c}\right)^{-1} e^{-\alpha x^{\beta}}\right]^{\delta v-v}\left(1+x^{c}\right)^{-k v-v} e^{-\alpha v x^{\beta}} \\
& \times\left(k c x^{c-1}+\left(1+x^{c}\right) \alpha \beta x^{\beta-1}\right)^{v} \\
& =\delta^{v} \sum_{i, j=0}^{\infty} \frac{(-1)^{i} \Gamma(\delta v-v+1)[\alpha(i+v)]^{j}}{\Gamma(\delta v-v+1-i) i ! j !}
\end{aligned}
$$




$$
\times x^{j \beta}\left(1+x^{c}\right)^{-k i-k v-v}\left(k c x^{c-1}+\left(1+x^{c}\right) \alpha \beta x^{\beta-1}\right)^{v} .
$$

Therefore,

$$
\begin{aligned}
\int_{0}^{\infty}\left[g_{E B W}(x)\right]^{v} d x & =\delta^{v} \sum_{i, j=0}^{\infty} \frac{(-1)^{i} \Gamma(\delta v-v+1)[\alpha(i+v)]^{j}}{\Gamma(\delta v-v+1-i) i ! j !} \\
& \times \int_{0}^{\infty} x^{j \beta}\left(1+x^{c}\right)^{-k i-k v-v}\left(k c x^{c-1}+\left(1+x^{c}\right) \alpha \beta x^{\beta-1}\right)^{v}
\end{aligned}
$$

Using binomial expansion

$$
\begin{aligned}
& \left(k c x^{c-1}+\left(1+x^{c}\right) \alpha \beta x^{\beta-1}\right)^{v} \\
& =\sum_{w=0}^{\infty}\left(\begin{array}{l}
v \\
w
\end{array}\right)\left[k c x^{c-1}\right]^{v-w}\left[\left(1+x^{c}\right) \alpha \beta x^{\beta-1}\right]^{w}
\end{aligned}
$$

we have

$$
\begin{aligned}
\int_{0}^{\infty}\left[g_{E B W}(x)\right]^{v} d x & =\delta^{v} \sum_{i, j=0}^{\infty} \frac{(-1)^{i} \Gamma(\delta v-v+1)[\alpha(i+v)]^{j}}{\Gamma(\delta v-v+1-i) i ! j !}\left(\begin{array}{l}
v \\
w
\end{array}\right)(\alpha \beta)^{w} k c^{v-w} \\
& \times \int_{0}^{\infty} x^{j \beta+c v-c w-+\beta w}\left(1+x^{c}\right)^{-k i-k v-v+w} d x
\end{aligned}
$$

Let $\mathrm{y}=\left(1+x^{c}\right)^{-1}$ then

$$
\begin{aligned}
& \int_{0}^{\infty}\left[g_{E B W}(x)\right]^{v} d x \\
& =\delta^{v} \sum_{i, j=0}^{\infty} \frac{(-1)^{i} \Gamma(\delta v-v+1)[\alpha(i+v)]^{j}}{\Gamma(\delta v-v+1-i) i ! j !}\left(\begin{array}{l}
v \\
w
\end{array}\right)(\alpha \beta)^{w} k c^{v-w} \frac{1}{c} \\
& \times \int_{0}^{\infty} y^{w-k i-k v-v-\frac{j \beta-c v+c w+v-\beta w}{c}-\frac{1}{c}-1}(1-y)^{\frac{j \beta+c v-c w-v+\beta w}{c}+\frac{1}{c}-1} d y \\
& =\delta^{v} \sum_{i, j=0}^{\infty} \frac{(-1)^{i} \Gamma(\delta v-v+1)[\alpha(i+v)]^{j}}{\Gamma(\delta v-v+1-i) i ! j !}\left(\begin{array}{l}
v \\
w
\end{array}\right)(\alpha \beta)^{w} k c^{v-w} \\
& \times \mathrm{B}\left(w-k i-k v-v-\frac{j \beta-c v+c w+v \beta w}{c}\right)-\frac{1}{c} \\
& \frac{j \beta+c v-c w-v+\beta w}{c}+\frac{1}{c}
\end{aligned}
$$

\subsection{Order Statistics}

The pdf of the $i^{\text {th }}$ order statistic and the corresponding moments are presented in this subsection. Suppose that $X_{1}, X_{2}, \ldots, X_{n}$ denote continuous independent EBW random variables with common cdf $\mathrm{G}(\mathrm{x})$ and pdf $\mathrm{g}(\mathrm{x})$, then the pdf of the $i^{\text {th }}$ order statistic for a random sample of size $\mathrm{n}$ is given by 


$$
\begin{aligned}
g_{i ; n}(x) & =\frac{n ! g(x)}{(i-1) !(n-i) !}[G(x)]^{n-1}[1-G(x)]^{n-i} \\
& =\frac{n !}{(i-1) !(n-i) !} \sum_{p=0}^{i-1}(-1)^{p}\left(\begin{array}{c}
n-i \\
p
\end{array}\right)\left[\left(1-\left(1+x^{c}\right)^{-k} e^{-\alpha x^{\beta}}\right)^{\delta}\right]^{i+p-1} g(x) .
\end{aligned}
$$

Using the binomial expansion,

$$
\begin{aligned}
& \left(1-\left(1+x^{c}\right)^{-k} e^{-\alpha x^{\beta}}\right)^{\delta} \\
& =\sum_{i, j=0}^{\infty} \frac{(-1)^{i} \Gamma(\delta(i+p-1)+1)}{j ! \Gamma(\delta(i+p-1)+1-j)}\left(\left(1+x^{c}\right)^{-k j} e^{-\alpha j x^{\beta}}\right)^{\delta},
\end{aligned}
$$

we have

$$
\begin{aligned}
g_{i ; n}(x) & =\frac{n ! g(x)}{(i-1) !(n-i) !} \sum_{p=0}^{i-1} \sum_{j=0}^{\infty} \frac{(-1)^{i} \Gamma(\delta(i+p-1)+1)}{j ! \Gamma(\delta(i+p-1)+1-j)}\left(\begin{array}{c}
n-1 \\
p
\end{array}\right) \\
& \times\left(\left(1+x^{c}\right)^{-k j} e^{-\alpha j x^{\beta}}\right)^{\delta}\left(1-\left(1+x^{c}\right)^{-k} e^{-\alpha x^{\beta}}\right)^{\delta-1} \\
& \times\left(1+x^{c}\right)^{-k-1} e^{-\alpha x^{\beta}}\left(k c x^{c-1}\left(1+x^{c}\right) \alpha \beta x^{\beta-1}\right) \\
& =\frac{n !}{(i-1) !(n-i) !} \sum_{p=0}^{i-1} \sum_{j=0}^{\infty} \frac{(-1)^{j} \delta \Gamma(\delta(i+p-1)+1)}{j ! \Gamma(\delta(i+p-1)+1-j)}\left(\begin{array}{c}
n-1 \\
p
\end{array}\right) \\
& \times\left(1+x^{c}\right)^{-(k+k j+1)} e^{-\alpha(j+1) x^{\beta}}\left(1-\left(1+x^{c}\right)^{-k} e^{-\alpha x^{\beta}}\right)^{\delta-1} \\
& \times\left(k c x^{c-1}\left(1+x^{c}\right) \alpha \beta x^{\beta-1}\right) .
\end{aligned}
$$

The $t$ th moment of the $i t h$ order statistic from the EBW distribution can be derived from the result of Barakat and Abdelkader [3].

$$
\mathrm{E}\left[X_{i ; n}^{t}\right]=\mathrm{t} \sum_{p=n+i-1}^{n}(-1)^{p-n+i-1}\left(\begin{array}{c}
p-1 \\
n-i
\end{array}\right)\left(\begin{array}{l}
n \\
p
\end{array}\right) \int_{0}^{\infty} x^{t-1}[1-G(x)]^{p} d x .
$$

Note that

$$
\begin{gathered}
\int_{0}^{\infty} x^{t-1}[1-G(x)]^{p} d x=\sum_{l=0}^{p}(-1)^{l}\left(\begin{array}{l}
p \\
l
\end{array}\right) \int_{0}^{\infty} x^{t-1}[G(x)]^{l} d x \\
=\sum_{l=0}^{p}(-1)^{l}\left(\begin{array}{l}
p \\
l
\end{array}\right) \int_{0}^{\infty} x^{t-1}\left[\left(1-\left(1+x^{c}\right)^{-k} e^{-\alpha x^{\beta}}\right)^{\delta}\right]^{l} d x \\
=\sum_{l=0}^{p} \sum_{s=0}^{\delta l}(-1)^{l+s}\left(\begin{array}{l}
p \\
l
\end{array}\right)\left(\begin{array}{c}
\delta l \\
s
\end{array}\right) \int_{0}^{\infty} x^{t-1}\left(\left(1+x^{c}\right)^{-k} e^{-\alpha x^{\beta}}\right)^{s} d x
\end{gathered}
$$




$$
\begin{aligned}
& =\sum_{l=0}^{p} \sum_{s=0}^{\delta l} \sum_{r=0}^{\infty}(-1)^{l+s+r} \frac{\left(\begin{array}{c}
p \\
l
\end{array}\right)\left(\begin{array}{c}
\delta l \\
s
\end{array}\right)(\alpha)^{r}(s)^{r}}{r ! c} \\
& \times \int_{0}^{1} y^{k s-\frac{r \beta}{c}-\frac{t}{c}-1}(1-y)^{\frac{r \beta+t}{c}-1} d y \\
& =\sum_{l=0}^{p} \sum_{s=0}^{\delta l} \sum_{r=0}^{\infty}(-1)^{l+s+r} \frac{\left(\begin{array}{l}
p \\
l
\end{array}\right)\left(\begin{array}{c}
\delta l \\
s
\end{array}\right)(\alpha)^{r}(s)^{r}}{r ! c} B\left(k s-\frac{r \beta}{c}-\frac{t}{c}, \frac{r \beta+t}{c}\right) .
\end{aligned}
$$

Therefore,

$$
\begin{aligned}
\mathrm{E}\left[X_{i ; n}^{t}\right] & =\mathrm{t} \sum_{p=n+i-1}^{n}(-1)^{p-n+i-1}\left(\begin{array}{l}
p-1 \\
n-i
\end{array}\right)\left(\begin{array}{l}
n \\
p
\end{array}\right) \int_{0}^{\infty} x^{t-1}[1-G(x)]^{p} d x \\
& =t \sum_{p=n+i-1}^{n} \sum_{l=0}^{p} \sum_{s=0}^{\delta l} \sum_{r=0}^{\infty}(-1)^{p-n+i+r+l+s-1} \frac{\left(\begin{array}{l}
p \\
l
\end{array}\right)\left(\begin{array}{c}
\delta l \\
s
\end{array}\right)\left(\begin{array}{l}
p-1 \\
n-i
\end{array}\right)\left(\begin{array}{l}
n \\
p
\end{array}\right)(\alpha)^{r}(s)^{r}}{r ! c} \\
& \times B\left(k s-\frac{r \beta}{c}-\frac{t}{c}, \frac{r \beta+t}{c}\right) .
\end{aligned}
$$

\subsection{L-moments}

The L moments introduced by Hoskings [13] are expectations of some linear combinations of order statistics and they exist whenever the mean of the distribution exits, even when some higher moments may not exist. They are relatively robust to the effects of outliers and are given by

$$
\lambda_{k+1}=\frac{1}{k+1} \sum_{j=0}^{k}(-1)^{j}\left(\begin{array}{l}
k \\
j
\end{array}\right) E\left(X_{K+1-j: k+1}\right), k=0,1,2, \ldots
$$

The L-moments of the EBW distribution can be readily obtained from equation (48). The first four $\mathrm{L}-$ moments are given by $\lambda_{1}=E X 1: 1,2=12 E X 2: 2-X 1: 2, \lambda_{3}=13 E X 3: 3-2 X 2: 3+X 1: 3$ and $\lambda 4=14 E X 4: 4-3 X 3: 4+3 X 2: 4-X 1: 4$, respectively.

\section{Estimation and Inference}

Maximum likelihood estimates (MLEs) of the parameters of the EBW distribution from complete samples are presented in this section. Suppose that $x 1,2, \ldots, x n$ denote the random sample of size $\mathrm{n}$ from EBW distribution and $\varphi=(c, k, \alpha, \beta, \delta) T$ denote the parameter vector. The log-likelihood function of the parameter vector is given by

$$
l=\log L=n \ln (\delta)+(\delta-1) \sum_{i=1}^{n} \ln \left(1-\left(1+x_{i}^{c}\right)^{-k} e^{-\alpha x_{i}^{\beta}}\right)
$$




$$
\begin{aligned}
& -(k+1) \sum_{i=1}^{n} \ln \left(1+x_{i}^{c}\right) \\
& -\alpha \sum_{i=1}^{n} x_{i}^{\beta}+\sum_{i=1}^{n} \ln \left(k c x_{i}^{c-1}+\left(1+x_{i}^{c}\right) \alpha \beta x_{i}^{\beta-1}\right) .
\end{aligned}
$$

The score vector is given by

$$
U_{n}(\varphi)=\left(\frac{\partial \mathrm{l}}{\partial \mathrm{c}}, \frac{\partial \mathrm{l}}{\partial \mathrm{k}}, \frac{\partial \mathrm{l}}{\partial \alpha}, \frac{\partial \mathrm{l}}{\partial \beta}, \frac{\partial \mathrm{l}}{\partial \delta}\right)^{T},
$$

and its elements are given by

$$
\begin{aligned}
& \frac{\partial \mathrm{l}}{\partial \mathrm{c}}=(\delta-1) \sum_{i=1}^{n} \frac{k\left(1+x_{i}^{c}\right)^{-k-1} e^{-\alpha x_{i}^{\beta} x_{i}^{c} \ln \left(x_{i}\right)}}{1-\left(1+x_{i}^{c}\right)^{-k} e^{-\alpha x_{i}^{\beta}}}-(\mathrm{k}+1) \sum_{i=1}^{n} \frac{x_{i}^{c} \ln \left(x_{i}\right)}{\left(1+x_{i}^{c}\right)} \\
& +\sum_{i=1}^{n} \frac{k x_{i}^{c-1}+k c x_{i}^{c-1} \ln \left(x_{i}\right)+\alpha \beta x_{i}^{\beta-1} x_{i}^{c} \ln \left(x_{i}\right)}{k c x_{i}^{c-1}+\left(1+x_{i}^{c}\right)^{-k} \alpha \beta x_{i}^{\beta}} \\
& \frac{\partial l}{\partial k}=(\delta-1) \sum_{i=1}^{n} \frac{k\left(1+x_{i}^{c}\right)^{-k} e^{-\alpha x_{i}^{\beta} \ln \left(1+x_{i}^{c}\right)}}{1-\left(1+x_{i}^{c}\right)^{-k} e^{-\alpha x_{i}^{\beta}}}-\sum_{i=1}^{n} \ln \left(1+x_{i}^{c}\right) \\
& +\sum_{i=1}^{n} \frac{c x_{i}^{c-1}}{k c x_{i}^{c-1}+\left(1+x_{i}^{c}\right)^{-k} \alpha \beta x_{i}^{\beta-1}} \\
& \frac{\partial l}{\partial \beta}=(\delta-1) \sum_{i=1}^{n} \frac{\left(1+x_{i}^{c}\right)^{-k} e^{-\alpha x_{i}^{\beta}} \alpha x_{i}^{\beta} \ln \left(x_{i}\right)}{1-\left(1+x_{i}^{c}\right)^{-k} e^{-\alpha x_{i}^{\beta}}}-\alpha \sum_{i=1}^{n} x_{i}^{\beta} \ln \left(x_{i}\right) \\
& +\sum_{i=1}^{n} \frac{\alpha\left(1+x_{i}^{c}\right) x_{i}^{\beta-1}\left(1+\beta \ln \left(x_{i}\right)\right)}{k c x_{i}^{c-1}+\left(1+x_{i}^{c}\right) \alpha \beta x_{i}^{\beta-1}} \\
& \frac{\partial l}{\partial \alpha}=(\delta-1) \sum_{i=1}^{n} \frac{\left(1+x_{i}^{c}\right)^{-k} x_{i}^{\beta} e^{-\alpha x_{i}^{\beta}}}{1-\left(1+x_{i}^{c}\right)^{-k} e^{-\alpha x_{i}^{\beta}}}-\sum_{i=1}^{n} x_{i}^{\beta} \\
& +\sum_{i=1}^{n} \frac{\left(1+x_{i}^{c}\right) x_{i}^{\beta-1}}{k c x_{i}^{c-1}+\left(1+x_{i}^{c}\right) \alpha \beta x_{i}^{\beta-1}}
\end{aligned}
$$

and

$$
\partial l \partial \delta=n \delta+i=1 n \ln \sigma(1-1+x i c-k) e-\alpha x i \beta,
$$

respectively. Solving the nonlinear system equations, $U n \varphi=0$ yields the maximum likelihood estimates. These equations can be obtained numerically via iterative methods such as Newton-Raphson technique using statistical software. 


\subsection{Asymptotic Confidence Intervals}

The Fisher information matrix of the EBW distribution is a $5 \times 5$ matrix given by $\mathrm{J}(\varphi)=$ $\left[\mathrm{J}_{\theta_{\mathrm{i}}, \theta_{\mathrm{j}}}\right]_{5 \times 5}=\mathrm{E}\left(-\frac{\partial^{2} \log \mathrm{L}}{\partial \theta_{\mathrm{i}} \partial \theta_{\mathrm{j}}}\right), \mathrm{i}, \mathrm{j}=1,2,3,4,5$. The toal Fisher information matrix $\mathrm{nJ}(\varphi)$ can be approximated by

$$
I_{n}(\varphi) \approx\left[-\left.\frac{\partial^{2} l}{\partial \theta_{\mathrm{i}} \partial \theta_{\mathrm{j}}}\right|_{\varphi=\widehat{\varphi}}\right]_{5 \times 5}, \mathrm{i}, \mathrm{j}=1,2,3,4,5
$$

For a given set of observations, we obtain the total Fisher information matrix (FIM) nJ( $\varphi$ ) after the convergence of the Newton-Raphson technique. Applying the usual large sample approximation, MLE of $\varphi$, that is $\widehat{\varphi}$ can be treated as being approximately $\mathrm{N}_{5}\left(\varphi, \mathrm{J}_{\mathrm{n}}(\varphi)^{-1}\right)$, where $\mathrm{J}_{\mathrm{n}}(\varphi)^{-1}$ is the expectation of FIM. When the conditions for parameters in the interior of the parameter space, but not on the boundary, are met, the asymptotic distribution becomes $\sqrt{\mathrm{n}}(\widehat{\varphi}-\varphi)$ is $\mathrm{N}_{5}\left(0, \mathrm{~J}(\varphi)^{-1}\right.$, where $J(\varphi)=\lim _{n \rightarrow \infty} n^{-1} I_{n}(\varphi)$ is the unit information matrix.This asymptotic behavior remains valid if the unit information matrix is replaced by the average sample information matrix evaluated at $\widehat{\varphi}$,say $\mathrm{n}^{1} \mathrm{I}_{\mathrm{n}}(\widehat{\varphi})$. The estimated asymptotic multivariate normal $\mathrm{N}_{5}\left(\varphi, \mathrm{I}_{\mathrm{n}}(\widehat{\varphi})^{-1}\right)$ distribution of $\widehat{\varphi}$ can be used to construct confidence intervals for the parameters. An $100(1-\xi) \%$ asymptotic confidence interval (ACI for each parameter $\varphi_{\mathrm{r}}$ is given by

$$
A C I_{r}=\left(\hat{\varphi}_{r}-z_{\frac{\xi}{2}} \sqrt{\hat{I}_{r r}}, \hat{\varphi}_{r}+z_{\frac{\xi}{2}} \sqrt{\hat{I}_{r r}}\right),
$$

Where $\hat{\mathrm{I}}_{\mathrm{rr}}$ is the $(\mathrm{r}, \mathrm{r})$ diagonal element of $\mathrm{I}_{\mathrm{n}}(\widehat{\varphi})^{-1}$ for $\mathrm{r}=1,2,3,4,5$ and $\mathrm{z}_{\frac{\xi}{2}}$ is the $1-\frac{\xi}{2}$ quantile of the standard normal distribution.

\subsection{Likelihood Ratio Test}

For a given dataset, the Likelihood ratio (LR) test can used to compare EBW distribution with its nested-models. For instance, to test $\mathrm{c}=\mathrm{k}=1$, the $\mathrm{LR}$ statistic is

$$
w=2[\ln (L((\hat{c}, \hat{k}, \hat{\alpha}, \hat{\beta}, \hat{\delta}))-\ln (L(1,1, \tilde{\alpha}, \tilde{\beta}, \tilde{\delta}))]
$$

where $\hat{c}, \hat{k}, \hat{\alpha}, \hat{\beta}$, and $\hat{\delta}$ are the unrestricted estimates, and $\widetilde{\alpha}, \tilde{\beta}$, and $\tilde{\delta}$ are the restricted estimates. The LR test rejects the null hypothesis if $\mathrm{w}>x_{\varepsilon}^{2}$, where $x_{\varepsilon}^{2}$ denote the upper $100 \in \%$ point of the $x^{2}$ distribution with 2 degrees of freedom.

\section{Simulation Study}


The accuracy and performance of the EBW distribution is investigated by conducting two simulations for different parameter values and sample sizes. The simulations were repeated $\mathrm{N}=1000$ times each with sample sizes $n=35, n=50, n=70, n=100, n=200, n=400$ and $n=800$ and the true parameters values $\mathrm{I}: \mathrm{c}=2.8, \mathrm{k}=1.2, \alpha=0.01, \beta=0.2, \delta=1.5$ and II $: \mathrm{c}=5.5, \mathrm{k}=7.5, \alpha=0.01, \beta=0.2$, $\delta=0.7$. Three quantities were computed in this simulation study: the mean, average bias and rootmean-square error. The mean estimate of the MLE $\hat{\varphi}$ of the parameter $\varphi=c, k, \alpha, \beta, \delta$ is given by

$$
\text { Mean }=\frac{\sum_{i=1}^{N} \hat{\varphi}_{i}}{N} .
$$

The root-mean-square error (RMSE) of the MLE $\hat{\varphi}$ of the parameter $\varphi=c, k, \alpha, \beta, \delta$ is given by

$$
R M S E=\sqrt{\frac{\sum_{i=1}^{N}\left(\hat{\varphi}_{i}-\varphi\right)^{2}}{N}} .
$$

The average bias of the MLE $\hat{\varphi}$ of the parameter $\varphi=c, k, \alpha, \beta, \delta$ is given by

$$
\operatorname{Bias}(\varphi)=\sqrt{\frac{\sum_{i=1}^{N}\left(\hat{\varphi}_{i}-\varphi\right)}{N}} .
$$

The mean of MLEs of the EBW distribution parameters along with their respective root-mean-square errors and average bias for different sample sizes are listed in Tables 3 and 4,

\begin{tabular}{|c|c|c|c|c|c|c|c|}
\hline \multirow[b]{2}{*}{ Parameter } & \multirow[b]{2}{*}{$\mathbf{n}$} & \multicolumn{3}{|c|}{$\mathbf{I}$} & \multicolumn{3}{|c|}{ II } \\
\hline & & Mean & Average Bias & RMSE & Mean & $\begin{array}{c}\text { Average } \\
\text { Bias }\end{array}$ & RMSE \\
\hline \multirow[t]{7}{*}{$\mathrm{c}$} & 35 & 3.2089 & 0.4089 & 6.9780 & 6.1635 & 0.6635 & 1.9732 \\
\hline & $\begin{array}{l}50 \\
70\end{array}$ & 3.1733 & $\begin{array}{l}0.3733 \\
0.3316\end{array}$ & 5.3093 & 5.9765 & 0.4765 & 1.7224 \\
\hline & & 3.1316 & 0.3316 & 1.0617 & 5.7721 & 0.2721 & $1 . 5 \longdiv { 0 4 1 }$ \\
\hline & 100 & 3.0820 & 0.2820 & 0.9990 & 5.6304 & 0.1304 & 1.2154 \\
\hline & 200 & 3.0309 & 0.2309 & 0.8730 & 5.5464 & 0.0464 & 0.9735 \\
\hline & 400 & 3.0268 & 0.2268 & 0.7993 & 5.5073 & 0.0073 & 0.7899 \\
\hline & 800 & 3.0230 & 0.2230 & 0.7300 & 5.5040 & 0.0040 & 0.6109 \\
\hline \multirow[t]{7}{*}{ k } & 35 & 1.4725 & 0.2725 & 0.7873 & 9.3832 & 1.8832 & 4.6803 \\
\hline & 50 & 1.4090 & 0.2090 & 0.7330 & 8.7313 & 1.2313 & 3.4260 \\
\hline & 70 & 1.4089 & 0.2089 & 0.7322 & 8.4000 & 0.9000 & 2.2979 \\
\hline & 100 & 1.3565 & 0.1565 & 0.6533 & 8.1310 & 0.6310 & 1.5389 \\
\hline & 200 & 1.2464 & 0.0464 & 0.5423 & 7.8630 & 0.3630 & 0.9900 \\
\hline & 400 & 1.1431 & -0.0569 & 0.4251 & 7.6610 & 0.1610 & 0.6500 \\
\hline & 800 & 1.1164 & -0.0836 & 0.3645 & 7.6055 & 0.1055 & 0.4643 \\
\hline \multirow[t]{7}{*}{$\alpha$} & 35 & 0.1252 & 0.1152 & 0.4548 & 0.2137 & 0.2037 & 1.5899 \\
\hline & 50 & 0.1245 & 0.1145 & 0.3826 & 0.6605 & 0.6505 & 1.3008 \\
\hline & 70 & 0.1219 & 0.1119 & 0.3557 & 0.1080 & 0.0980 & 0.3840 \\
\hline & 100 & 0.1108 & 0.1008 & 0.3564 & 0.0976 & 0.0876 & 0.3201 \\
\hline & 200 & 0.0720 & 0.0620 & 0.2403 & 0.0661 & 0.0561 & 0.2216 \\
\hline & 400 & 0.0394 & 0.0294 & 0.1423 & 0.0385 & 0.0285 & 0.1295 \\
\hline & 800 & 0.0233 & 0.0133 & 0.0453 & 0.0218 & 0.0118 & 0.0344 \\
\hline \multirow[t]{7}{*}{$\beta$} & 35 & 0.3906 & 0.1906 & 0.6030 & 1.3533 & 1.1533 & 21.4730 \\
\hline & 50 & 0.3698 & O. 1698 & 0.5049 & 0.8816 & 0.6816 & 5.7462 \\
\hline & 70 & 0.3690 & 0.1690 & 0.4711 & 0.4778 & 0.2778 & 0.5709 \\
\hline & 100 & 0.3596 & 0.1596 & 0.3750 & 0.4008 & 0.2008 & 0.3824 \\
\hline & 200 & 0.3325 & 0.1325 & 0.2403 & 0.2964 & 0.0964 & 0.2277 \\
\hline & 400 & 0.3038 & 0.1038 & 0.1745 & 0.2434 & 0.0434 & 0.1049 \\
\hline & 800 & 0.2990 & 0.0990 & 0.1494 & 0.2424 & 0.0424 & 0.2282 \\
\hline \multirow[t]{7}{*}{$\delta$} & 35 & 3.2622 & 1.7622 & 8.3689 & 1.7313 & 1.0313 & 8.3791 \\
\hline & 50 & 2.5243 & 1.0243 & 7.2240 & 1.6468 & 0.9468 & 8.2344 \\
\hline & 70 & 2.3602 & 0.8602 & 4.8890 & 1.4017 & 0.7017 & 4.6477 \\
\hline & 100 & 1.8614 & 0.7833 & 4.5192 & 1.3096 & 0.6096 & 3.7003 \\
\hline & 200 & 1.8614 & 0.3614 & 2.4956 & 1.0655 & 0.3655 & 2.6472 \\
\hline & 400 & 1.6217 & 0.1217 & 2.1650 & 0.8689 & 0.1689 & 1.4403 \\
\hline & 800 & 1.4967 & -0.0033 & 0.2755 & 0.7434 & 0.0434 & 0.2282 \\
\hline
\end{tabular}
respectively.

Table 3. Monte Carlo Simulation Results: Mean, Average Bias and RMSE

For Table 4, N $=1000$ samples are simulated for the true parameters values I : c $=0.9, \mathrm{k}=1.0, \alpha=$ $2.0, \beta=4.8, \delta=1.0$ and II : $\mathrm{c}=1.0, \mathrm{k}=0.9, \alpha=2.4, \beta=5.0, \delta=0.8$. 
Table 4. Monte Carlo Simulation Results: Mean, Average Bias and RMSE

\begin{tabular}{|c|c|c|c|c|c|c|c|}
\hline \multirow[b]{2}{*}{ Parameter } & \multirow[b]{2}{*}{$\mathbf{n}$} & \multicolumn{3}{|c|}{$\mathbf{I}$} & \multicolumn{3}{|c|}{ II } \\
\hline & & Mean & Average Bias & RMSE & Mean & Average Bias & RMSE \\
\hline \multirow[t]{7}{*}{ c } & 35 & 1.1323 & 0.2323 & 0.9756 & 1.0245 & 0.0245 & 0.8598 \\
\hline & 50 & 0.9665 & 0.0665 & 0.8105 & 0.9159 & -0.0841 & 0.7577 \\
\hline & 70 & 0.9377 & 0.0377 & 0.7519 & 0.9094 & -0.0906 & 0.6907 \\
\hline & 100 & 0.9339 & 0.0339 & 0.7135 & 0.8488 & -0.1512 & 0.6344 \\
\hline & 200 & 0.9108 & 0.0108 & 0.6240 & 0.8391 & -0.1609 & 0.5786 \\
\hline & 400 & 0.9080 & 0.0080 & 0.5213 & 0.8285 & -0.1715 & 0.4926 \\
\hline & 800 & 0.9034 & 0.0034 & 0.4210 & 0.7966 & -0.2034 & 0.4016 \\
\hline \multirow[t]{7}{*}{$\mathbf{k}$} & 35 & 2.3253 & 1.3253 & 2.4019 & 2.3655 & 1.4655 & 2.4185 \\
\hline & 50 & 2.2319 & 1.2319 & 2.2713 & 2.2922 & 1.3922 & 2.2761 \\
\hline & 70 & 2.1641 & 1.1641 & 2.1877 & 2.0913 & 1.1913 & 2.1820 \\
\hline & 100 & 2.0618 & 1.0618 & 2.0689 & 2.0811 & 1.1811 & 2.1009 \\
\hline & 200 & 1.8112 & 0.8112 & 1.7352 & 1.8381 & 0.9381 & 1.7031 \\
\hline & 400 & 1.6082 & 0.6082 & 1.3569 & 1.4795 & 0.5795 & 1.2065 \\
\hline & 800 & 1.2991 & 0.2991 & 0.8822 & 1.1876 & 0.2876 & 0.7222 \\
\hline \multirow{7}{*}{$\alpha$} & 35 & 2.1451 & 0.1451 & 1.3001 & 8.5739 & 6.1739 & 1.9055 \\
\hline & 50 & 2.1343 & 0.1343 & 0.7557 & 2.8089 & 0.4089 & 1.4892 \\
\hline & 70 & 2.1202 & 0.1202 & 0.6079 & 2.6653 & 0.2653 & 0.7914 \\
\hline & 100 & 2.0987 & 0.0987 & 0.5165 & 2.6368 & 0.2368 & 0.6541 \\
\hline & 200 & 2.0788 & 0.0788 & 0.4043 & 2.5742 & 0.1742 & 0.4586 \\
\hline & 400 & 2.0506 & 0.0506 & 0.3106 & 2.5173 & 0.1173 & 0.3452 \\
\hline & 800 & 2.0228 & 0.0228 & 0.2513 & 2.4615 & 0.0615 & 0.2585 \\
\hline \multirow[t]{7}{*}{$\beta$} & 35 & 7.8893 & 3.0893 & 9.9513 & 5.3899 & 0.3899 & 23.0224 \\
\hline & 50 & 6.0169 & 1.2169 & 3.7305 & 5.0083 & 0.0083 & 3.0644 \\
\hline & 70 & 5.4835 & 0.6835 & 1.9066 & 5.1654 & 0.1654 & 2.1063 \\
\hline & 100 & 5.1948 & 0.3948 & 1.3406 & 5.5817 & 0.5817 & 1.2072 \\
\hline & 200 & 4.9924 & O. 1924 & 0.9699 & 4.9505 & -0.0495 & 0.9024 \\
\hline & 400 & 4.8506 & 0.0506 & 0.5796 & 4.9221 & -0.0779 & 0.7615 \\
\hline & 800 & 4.8251 & 0.0251 & 0.5741 & 4.7966 & -0.2034 & 0.4016 \\
\hline \multirow[t]{7}{*}{8} & 35 & 11.3895 & 10.3895 & 36.4545 & 9.5353 & 8.7353 & 31.9856 \\
\hline & 50 & 10.0516 & 9.0516 & 30.6932 & 8.4925 & 7.6925 & 27.7475 \\
\hline & 70 & 9.8992 & 8.8992 & 28.5762 & 8.2898 & 7.4898 & 26.1824 \\
\hline & 100 & 9.2090 & 8.2090 & 25.1325 & 7.2969 & 6.4969 & 23.1872 \\
\hline & 200 & 6.3469 & 5.3469 & 18.6272 & 5.0284 & 4.2284 & 14.5848 \\
\hline & 400 & 3.7574 & 2.7574 & 9.8552 & 2.6604 & 1.8604 & 6.1662 \\
\hline & 800 & 2.0094 & 1.0094 & 4.6033 & 1.4015 & 0.6015 & 2.5003 \\
\hline
\end{tabular}

From the results in Tables 3 and 4, it can be verified that the mean of MLEs converges to the true value and the RMSEs decay toward zero as the sample size $\mathrm{n}$ increases. Also, the average bias decrease as the sample size $\mathrm{n}$ increases for all parametric values.

\section{Applications}

In this section, applications of the proposed model to real datasets are presented. The first dataset is used to illustrate that EBW distribution can be a better fit when compared with its sub-models (EB, BXII, ELLoG, LLoG and Exponential (Exp) distributions) as well as with the non-nested Exponentiated Kumaraswamy Weibull (EKW) distribution which was used by Huang and Oluyede [12]. The pdf of EKW distribution is given by

$$
\begin{aligned}
& g_{E K W}(x ; a, b, c, \theta, \lambda)=a b c \theta \lambda^{c} x^{c-1} e^{-(\lambda \mathrm{x})^{c}}\left(1-e^{-(\lambda \mathrm{x})^{c}}\right)^{a-1} \\
& \left(1-\left[1-e^{-(\lambda \mathrm{x})^{c}}\right]^{a}\right)^{b-1} \times\left[1-\left\{1-\left[1-e^{-(\lambda \mathrm{x})^{c}}\right]^{a}\right\}^{b}\right]^{\theta-1} .
\end{aligned}
$$

Tables 6 and 8 lists the maximum likelihood estimates (MLEs) of the parameters and standard errors (in parenthesis) for the fitted EBW, EB, BXII, ELLoG, LLoG, Exp, EKD and EKW distributions and the values of the following statistics : Akaike Information Criterion $(\mathrm{AIC}=2 \mathrm{p}-2 \log (\mathrm{L}))$, Corrected Akaike Information Criterion $\left(\operatorname{AICC}=A I C+\frac{2 p(p+1)}{n-p-1}\right)$, Bayesian Information Criterion $(\mathrm{BIC}=\mathrm{p} \log (\mathrm{n})-2 \log (\mathrm{L})$ ), Hannan-Quinn Information Criterion (HQIC = $2 \log (\mathrm{L})+2 \mathrm{p} \log (\log (\mathrm{n})))$, where $\mathrm{L}=\mathrm{L}(\hat{\varphi})$ denote the value of the likelihood function evaluated at the parameter estimates, $\mathrm{n}$ represent the number of observations and $\mathrm{p}$ represent the number of estimated parameters. Furthermore, the goodness-of-fit statistics : Cramer-von-Mises $\left(w^{*}\right)$ and 
Anderson-Darling $\left(A^{*}\right)$ are also presented. The smaller the goodness-of-fit statistics the better the fit. The computations were performed using R software.

\subsection{Breaking Stress Data}

The data set given in Table 5 represent breaking stress of carbon fibres (Gba) reported in Nichols and Padgett [21]. The dataset consist of 100 observations. Initial values for EBW distribution in R code are taken to be : $\mathrm{c}=10, \mathrm{k}=0.0001, \delta=8, \alpha=0.1, \beta=1.5$.

Table 5. Breaking stress of carbon fibres (in Gba) data

\begin{tabular}{llllllllllllll}
\hline 3.70 & 2.74 & 2.73 & 2.50 & 3.60 & 3.11 & 3.27 & 2.87 & 1.47 & 3.11 & 4.42 & 2.41 & 3.19 & 3.22 \\
1.69 & 3.28 & 3.09 & 1.87 & 3.15 & 4.90 & 3.75 & 2.43 & 2.95 & 2.97 & 3.39 & 2.96 & 2.53 & 2.67 \\
2.93 & 3.22 & 3.39 & 2.81 & 4.20 & 3.33 & 2.55 & 3.31 & 3.31 & 2.85 & 2.56 & 3.56 & 3.15 & 2.35 \\
2.55 & 2.59 & 2.38 & 2.81 & 2.77 & 2.17 & 2.83 & 1.92 & 1.41 & 3.68 & 2.97 & 1.36 & 0.98 & 2.76 \\
4.91 & 3.68 & 1.84 & 1.59 & 3.19 & 1.57 & 0.81 & 5.56 & 1.73 & 1.59 & 2.00 & 1.22 & 1.12 & 1.71 \\
2.17 & 1.17 & 5.08 & 2.48 & 1.18 & 3.51 & 2.17 & 1.69 & 1.25 & 4.38 & 1.84 & 0.39 & 3.68 & 2.48 \\
0.85 & 1.61 & 2.79 & 4.70 & 2.03 & 1.80 & 1.57 & 1.08 & 2.03 & 1.61 & 2.12 & 1.89 & 2.88 & 2.82 \\
2.05 & 3.65 & & & & & & & & & & & & \\
\hline
\end{tabular}

Table 6. Estimation of Models for breaking stress of carbon fibres (in Gba) data

\begin{tabular}{|c|c|c|c|c|c|c|c|c|c|c|c|c|}
\hline \multicolumn{6}{|c|}{ Estimates } & \multicolumn{7}{|c|}{ Statistics } \\
\hline Model & c & $\mathrm{k}$ & $\alpha$ & $\beta$ & $\delta$ & $-2 \log \mathrm{L}$ & AIC & AICC & BIC & $\mathrm{HIQC}$ & $w^{*}$ & $A^{*}$ \\
\hline \multirow[t]{2}{*}{ EBW } & 32.602 & 0.0019088 & 0.098987 & 2.5746 & 1.2187 & 282.5159 & 292.5159 & 293.1542 & 305.5417 & 297.7877 & 0.06467943 & 0.4062228 \\
\hline & $(0.00024515)$ & $(0.0046326)$ & $(0.080349)$ & $(0.70248)$ & $(0.52217)$ & & & & & & & \\
\hline \multirow[t]{2}{*}{ EB } & 5.696500 & 0.112883 & - & - & 0.527553 & 864.087 & 8700.08 & 870.33 & 877.8955 & 873.243 & 0.8637942 & 4.852515 \\
\hline & $(1.267685)$ & $(0.027122)$ & - & - & $(0.035251)$ & & & & & & & \\
\hline \multirow[t]{2}{*}{ BXII } & 5.939512 & 0.187414 & - & - & - & 378.9669 & 382.9669 & 383.0906 & 6388.1772 & 385.0756 & 0.872757 & 4.902512 \\
\hline & $(1.279319)$ & $(0.043787)$ & - & - & - & & & & & & & \\
\hline \multirow[t]{2}{*}{ ELLoG } & 0.910017 & - & - & - & 0.523430 & 1005.831 & 1009.954 & 1015.041 & 1015.041 & 1011.939 & 0.2937121 & 1.575019 \\
\hline & $(0.076288)$ & - & - & - & $(0.05287)$ & & & & & & & \\
\hline \multirow[t]{2}{*}{ LLoG } & 1.62874 & - & - & - & - & 467.6428 & 469.6428 & 469.6836 & 472.2479 & 470.6971 & 0.3644871 & 1.93926 \\
\hline & $(0.12877)$ & - & - & - & - & & & & & & & \\
\hline \multirow[t]{3}{*}{ Exp } & - & - & 0.3814764 & - & - & 392.7417 & 394.7417 & 394.7825 & 397.3469 & 95.7961 & 0.07182727 & 0.4117877 \\
\hline & - & - & $(0.021193)$ & - & - & & & & & & & \\
\hline & $\hat{c}$ & $\hat{a}$ & $\hat{b}$ & $\hat{\theta}$ & $\hat{\lambda}$ & & & & & & & \\
\hline \multirow[t]{2}{*}{ EKW } & 1.584154 & 2.525451 & 6.457514 & 0.797712 & 0.239382 & 282.6165 & 292.6165 & 293.548 & 305.6423 & 297.8883 & 0.06875902 & 0.4048473 \\
\hline & $(0.75113)$ & $(0.0011470)$ & $(0.25570)$ & $(0.00088177)$ & $(0.27763)$ & & & & & & & \\
\hline
\end{tabular}


The asymptotic covariance matrix of the MLE's for the EBW distribution, $I_{N}^{-1}(\hat{\varphi})$ is given by :

$\left(\begin{array}{lllcr}0.0000000900964 & 0.0000003859855 & -0.0001225090 & -000001954973 & 0.0001716851 \\ 0.0000003859855 & 0.00002146137 & -0.0004235051 & -0.0001571949 & 0.0012282757 \\ -0.0001225090 & -0.0004235051 & 0.2726660110 & 0.03932091 & -0.3416936879 \\ -0.00001954973 & -0.0001571949 & 0.0393209125 & 0.006455961 & -0.0560270381 \\ -0.0001716851 & 0.001228276 & -0.341693687 & -0.05602704 & 0.4934774847\end{array}\right)$

and the approximate 95\% two-sided confidence interval for the model parameters $\mathrm{c}, \mathrm{k}, \alpha, \beta$ and $\delta$ are given by $32.60 \pm 0.0004804857,0.0019088 \pm 0.0090799783,0.098987 \pm 0.1574840350$, $2.5746 \pm 1.3768598713$, and $1.2185 \pm 1.37685987$, respectively.

The LR statistics for testing the hypothesis $H_{0}$ : EB against $H_{a}$ : EBW, $H_{0}$ : BXII against $H_{a}$ : EBW, $H_{0}$ : ELLoG against $H_{a}$ : EBW, $H_{0}$ : LLoG against $H_{a}$ : EBW and $H_{0}$ : Exp against $H_{a}$ : EBW are 581.5711 (pvalue<0.0001), 96.451 (pvalue<0.0001), 723.3151 (pvalue<0.0001), 185.1269 (pvalue <0.0001) and 110.2258 (pvalue<0.0001), respectively. According to the LR statistics the EBW distribution fits better than the five sub-models. For model selection, EBW distribution compares favorably with the EKW distribution since the AIC, AICC, BIC and HIOC statistics for EBW and EKW distributions are very close. The smallest values of both the $w^{*}$ and $A^{*}$ show that the EBW distribution fit better than the nested-models and the non-nested models for breaking stress data. Consequently, the EBW distribution is the best fitted distribution when compared to the nested and non-nested distributions. Plots of the fitted densities are given in Figure 7.

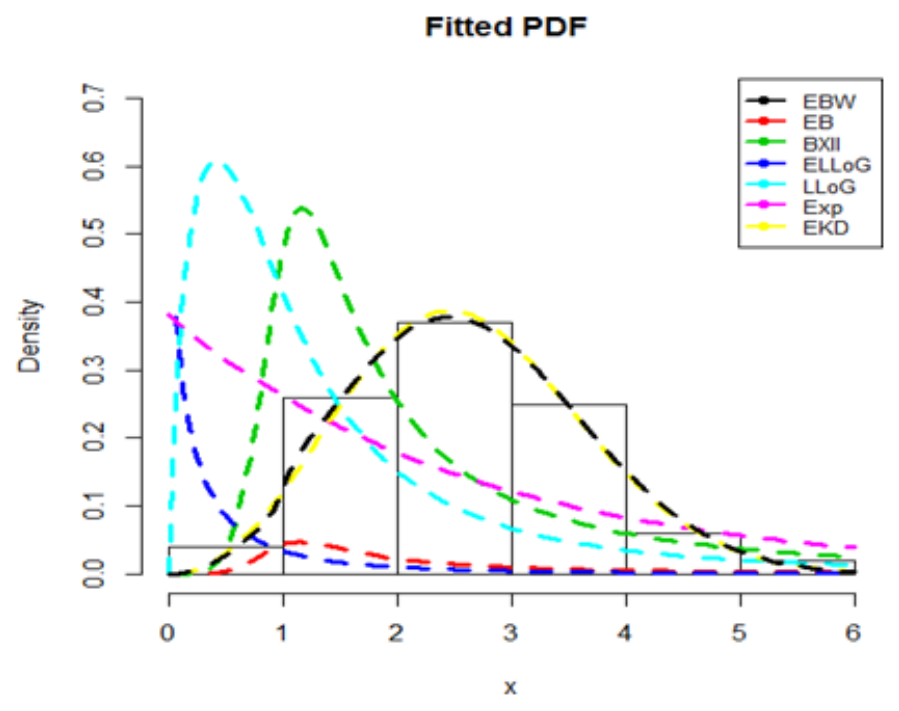

Figure 7. Fitted PDF for breaking stress of carbon fibres (in Gba) data 


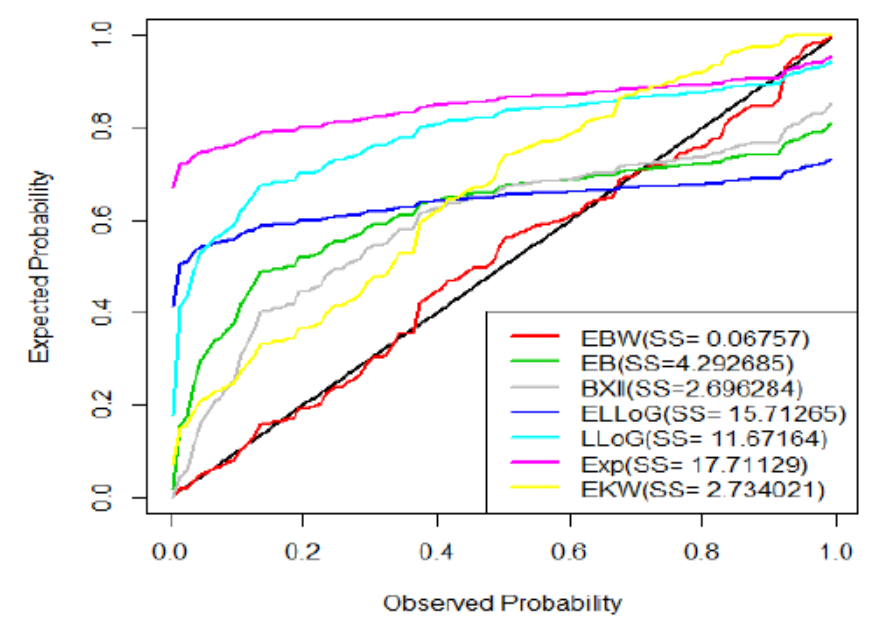

Figure 8. Probability Plots for breaking stress of carbon fibres (in Gba) data

\subsection{Air Conditioning System of an Airplane Data}

The dataset taken from Linhart and Zucchini [15] represents failure times of the air conditioning system of an airplane (see Proschan [26]). Initial values for EBW distribution in R code are $\mathrm{c}=10, \mathrm{k}=$ $0.0001, \delta=8, \alpha=0.1, \beta=1.5$. This dataset is used to illustrate that fact that the EBW distribution is a "better"fit when compared with its sub-models (EB, BXII, ELLoG, LLoG and Exponential (Exp) distributions) and the non-nested EKD distribution (Huang and Oluyede [12]). The pdf of EKD distribution is given by

$$
\begin{aligned}
& g_{E K W}(x ; \alpha, \lambda, \delta, \varnothing, \theta)=a \lambda \delta \varnothing \theta x^{-\delta-1}\left(1-\lambda \mathrm{x}^{-\delta}\right)^{\alpha-1} \\
& \quad\left[1-\left(1-\lambda \mathrm{x}^{-\delta}\right)^{-\alpha}\right]^{\theta-1} \times\left\{1-\left[1-\left(1-\lambda \mathrm{x}^{-\delta}\right)^{-\alpha}\right]^{\emptyset}\right\}^{\theta-1},
\end{aligned}
$$

for $\alpha, \lambda, \delta, \emptyset, 0>0$.

Table 7. Failure Times of the Air Conditioning System of an Airplane Data

\begin{tabular}{lllllllllllll}
\hline 5.50 & 5.00 & 4.90 & 6.40 & 5.10 & 5.20 & 5.20 & 5.00 & 4.70 & 4.00 & 4.50 & 4.20 & 4.10 \\
4.56 & 5.01 & 4.70 & 3.13 & 3.12 & 2.68 & 2.77 & 2.70 & 2.36 & 4.38 & 5.73 & 4.35 & 6.81 \\
1.91 & 2.66 & 2.61 & 1.68 & 2.04 & 2.08 & 2.13 & 3.80 & 3.73 & 3.71 & 3.28 & 3.90 & 4.00 \\
3.80 & 4.10 & 3.90 & 4.05 & 4.00 & 3.95 & 4.00 & 4.50 & 4.50 & 4.20 & 4.55 & 4.65 & 4.10 \\
4.25 & 4.30 & 4.50 & 4.70 & 5.15 & 4.30 & 4.50 & 4.90 & 5.00 & 5.35 & 5.15 & 5.25 & 5.80 \\
5.85 & 5.90 & 5.75 & 6.25 & 6.05 & 5.90 & 3.60 & 4.10 & 4.50 & 5.30 & 4.85 & 5.30 & 5.45 \\
5.10 & 5.30 & 5.20 & 5.30 & 5.25 & 4.75 & 4.50 & 4.20 & 4.00 & 4.15 & 4.25 & 4.30 & 3.75 \\
3.95 & 3.51 & 4.13 & 5.40 & 5.00 & 2.10 & 4.60 & 3.20 & 2.50 & 4.10 & 3.50 & 3.20 & 3.30 \\
4.60 & 4.30 & 4.30 & 4.50 & 5.50 & 4.60 & 4.90 & 4.30 & 3.00 & 3.40 & 3.70 & 4.40 & 4.90 \\
4.90 & 5.00 & & & & & & & & & & & \\
\hline
\end{tabular}


Table 8. Estimation of Models for Failure Times of the Air Conditioning System of an Airplane Dataset.

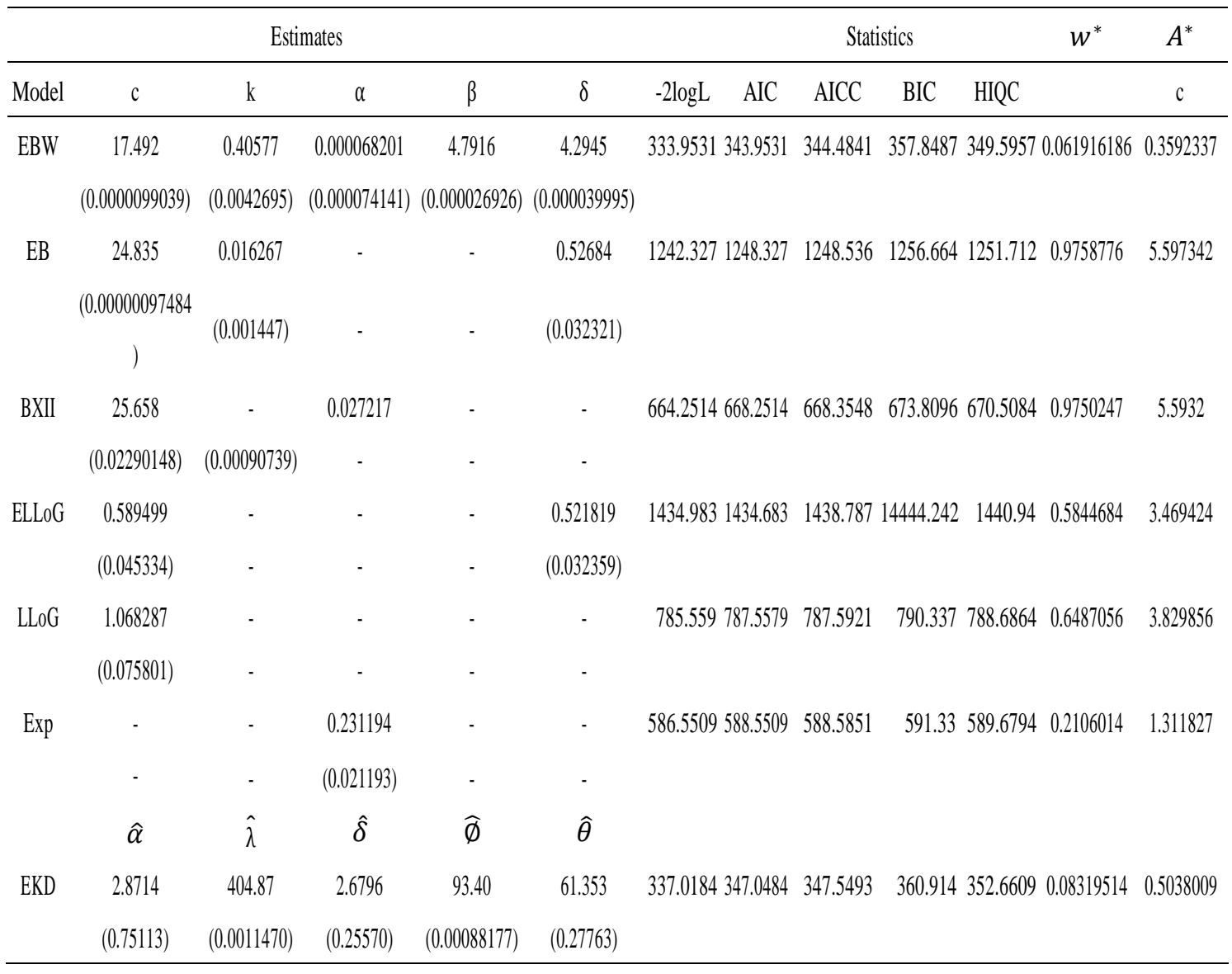

The asymptotic covariance matrix of the MLE's for the EBW distribution, $I_{n}^{-1}(\hat{\varphi})$ is given by :

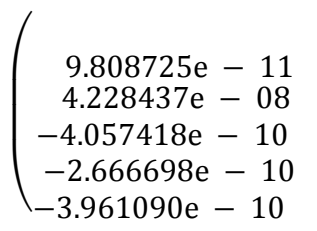

$$
\begin{array}{r}
4.228437 e-08 \\
1.822834 e-05 \\
-1.749110 e-07 \\
-1.149585 e-07 \\
-1.707584 e-07
\end{array}
$$

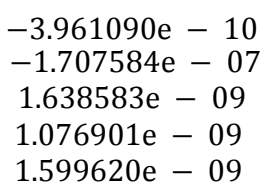
$-4.057418 \mathrm{e}-10$
$-1.749110 \mathrm{e}-07$
$5.496825 \mathrm{e}-09$
$1.107670 \mathrm{e}-09$
$1.638583 e-09$
$-2.666698 \mathrm{e}-10$
$-1.149585 \mathrm{e}-07$
$1.107670 \mathrm{e}-09$
$7.250006 e-10$
$1.076901 \mathrm{e}-09$

and the approximate $95 \%$ two-sided confidence interval for the parameters $\mathrm{c}, \mathrm{k}, \alpha, \beta$ and $\delta$ are given by $17.491 \pm 0.0004804857,0.40577 \pm 0.0090799783,0.000068201 \pm 0.1574840350,4.7916 \pm$ $1.3768598713,4.2945 \pm 1.0234616494$, respectively. The LR statistics for testing the hypothesis $\mathrm{H}_{0}$ : EB against $\mathrm{H}_{\mathrm{a}}$ : EBW, $\mathrm{H}_{0}$ : BXII against Ha: EBW, $\mathrm{H}_{0}$ : ELLoG against $\mathrm{H}_{\mathrm{a}}$ : EBW, $\mathrm{H}_{0}$ : LLoG against $\mathrm{H}_{\mathrm{a}}$ : EBW and $\mathrm{H}_{0}$ : Exp against $\mathrm{H}_{\mathrm{a}}$ : EBW are 108.3739 (pvalue $<0.0001$ ), 330.2983 (p-value<0.0001),1100.73 (p-value<0.0001), 453.609 (p-value<0.0001) and 252.5973 (p-value $<0.0001$ ), respectively. We conclude that the EBW distribution is a significantly better than the EB, BXII, ELLoG, LLoG and Exp distributions. The smaller values of AIC, AICC, HQIC and BIC statistics gives clear evidence that EBW distribution fits better when compared with the nested and non-nested models. The EBW distribution is the "better"model since it has the smallest value for the goodness-of-fit statistics $w^{*}$ and $A^{*}$, respectively. Plots of the fitted densities are given in Figure 9. 
Figure 9. Fitted PDF for Air Conditioning System of an Airplane data

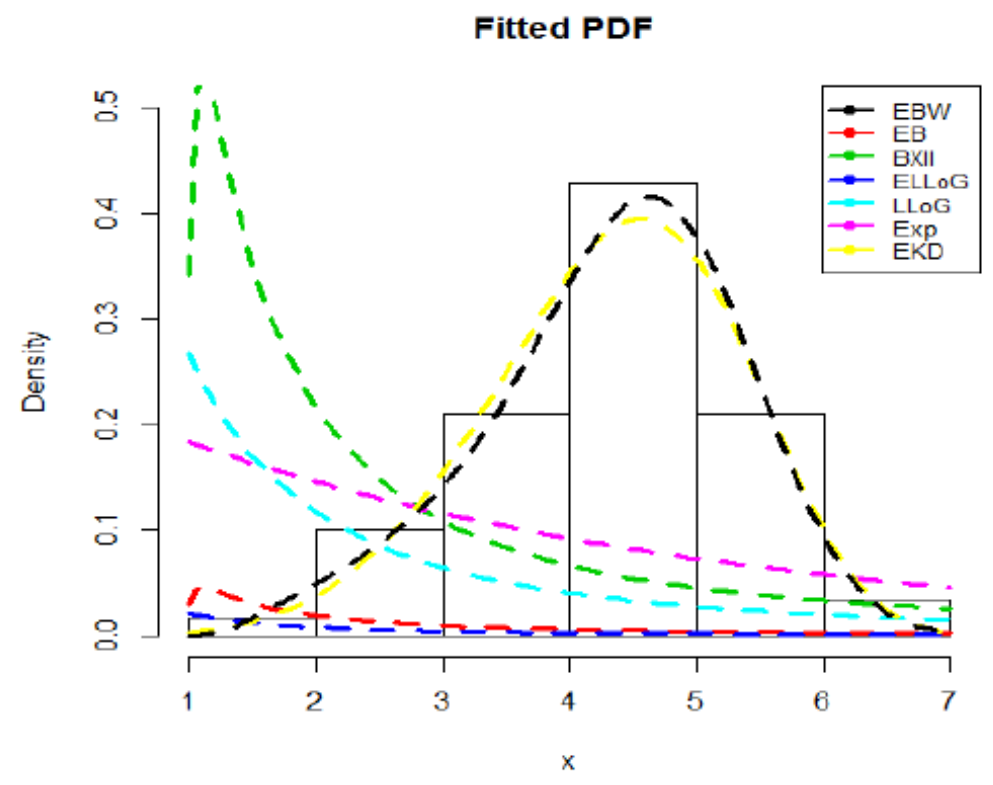

\section{Concluding Remarks}

A new and generalized distribution called the exponentiated Burr XII Weibull (EBW) distribution has been proposed and studied. The EBW distribution has several new and well known distributions including the Burr XII, Lomax Exponential, Lomax Rayleigh, Lomax, Weibull, Rayleigh, Exponentiated Weibull, Exponentiated Rayleigh and Exponentiated exponential distributions as special cases. The EBW distribution possesses hazard function with very useful and flexible behavior. We also obtain closed form expressions for the moments, conditional moments, mean and median deviations, Bonferroni and Lorenz curves, distribution of order statistics and R'enyi entropy. Maximum likelihood estimation technique is used to estimate the model parameters. Finally, the EBW distribution is fitted to real datasets in order to illustrate its applicability and usefulness.

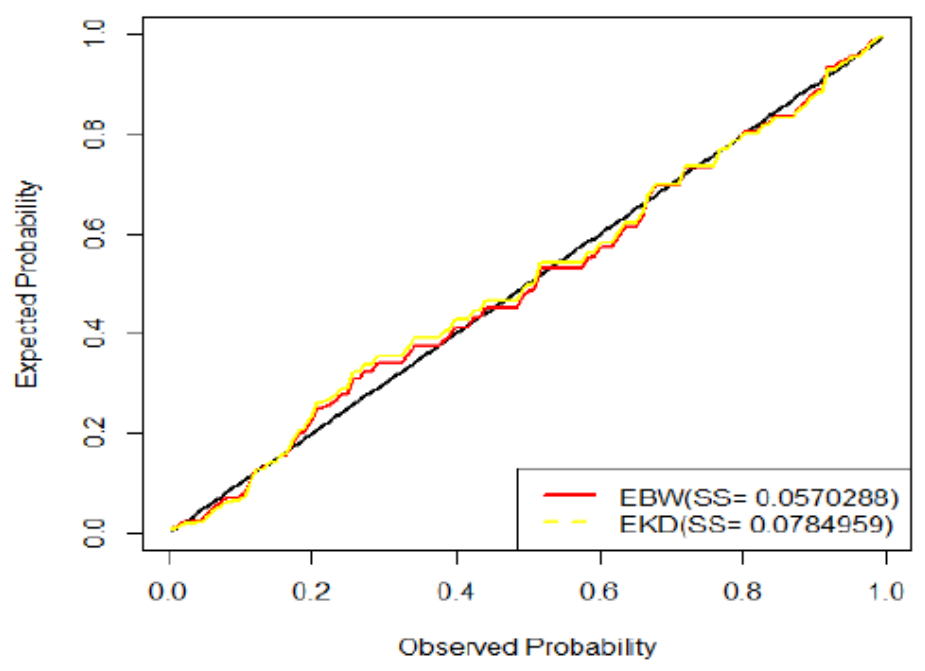

Figure 10. Probability Plots for Air Conditioning System of an Airplane data 


\section{References}

[1] Abdul-Moniem, I. B. and Abdel-Hameed, H. F. (2012). On Exponentiated Lomax Dis- tribution. IJMA, 3(5), 2144-2150.

[2] Abd-Elfattah, A. M., Hassan, A.S. and Nassr, S. G. (2008). Estimation in Step-Stress Partiallt Accelerated Life Tests for the Burr XII Distribution Using Type I Censoring. Statistical Methology, $5(6), 502-514$.

[3] Barakat, H. and Abdelkader, Y. (2014). Computing the Moments of Order Statistics from Nonidentical

Random Variables. Stat. Meth. Appl, 13, 15-26.

[4] Burr, I. W. (1942). Cumulative Frequency Functions. Annals of Mathematical Statistics, 13, 215232.

[5] Burr, I. W. and Cislak,P. J. (1968). On a General Systems of Distributions: I. Its Curve-Shaped Characteristic, II. The Sample Median. Journal of American Statistcal Assocaition, 63, 627-635.

[6] Gupta, R. C., Gupta, P. L. and Gupta, R. D. (1998). Modeling Failure Time Data by Lehmann Alternatives. Communications in Statistics - Theory and Methods, 27, 887-904.

[7] Gurvich,M.R.,DiBenedetto,A. T.and Ranade,S. V. (1997). A New Statistical Distribution for Characterizing the Random Strength of Brittle Materials. Journal of Materials Science, 32, 25592564.

[8] Hamid, A.H. (2009). Constant-Partially Accelerated Life Tests for Burr XII Distribution with Progressive Type II Censoring. Computational Statistics and Data Analysis, 53(7), 2511-2523.

[9] Hatke, M. A. (1949). A Certain Cumulative Probability Function. The Annals of Math- ematical Statistics, 20, 461-463.

[10] Haupt, E. and Schabe, H. (1992). A New Model for A Lifetime Distribution With Bathtub Shaped Failure Rate. Microelectronics and Reliability, 32, 633-639.

[11] Hjorth, U. (1980). A Reliability Distribution with Increasing, Decreasing, Constant and Bathtub-Shaped Failure Rates. Technometrics, 22(1), 99-107.

[12] Huang, S. and Oluyede, B. O. (2014). Exponentiated Kumaraswamy Dagum Distribution with Applications to Income and Lifetime Data. Journal of Statistical Distributions and Applications, 1(8), $1-20$. 
[13] Hoskings, J. R. M. (1990). L-Moments: Analysis and Estimation of Distributions Using Linear Combinations of Order Statistics. Journal of the Royal Statistical Society, B 52, 105-124.

[14] Lewis, A. W. (1981). The Burr Distribution as a General Parametric Family in Survivor- ship and Reliability Theory Applications. Ph.D. Thesis, University of North Carolina of Chapel Hill.

[15] Linhart, H. and Zucchini, W. (1986). Model Selection. New York.

[16] Mdlongwa, P., Oluyede, B. O., Amey, A. and Huang, S. (2017). (2014), The Burr XII Modified Weibull Distribution: Model, Properties and Applications. Electronic Journal of Applied Statistical Analysis, 10(1), 118-145.

[17] Mudholkar, G. S. and Hutson, A.D. (1996). The Exponentiated Weibull Family: Some Properties and a Flood Data Application. Communications in Statistics-Theory and Methods, 25, 3059-3083.

[18] Mudholkar, G. S. and Srivastava, D. K. (1993). Exponentiated Weibull Family for Analyzing Bathtub Failure-rate Data. IEEE Transactions on Reliability, 42(2), 299-302.

[19] Mudholkar, G. S., Srivastava, D. K. and Freimer, M. (1995). The exponentiated Weibull family: A Reanalysis of the Bus Motor Failure Data. Technometrics, 37 (4): 436-445.

[20] Nelson, W. (1982). Lifetime Data Analysis. Wiley, New York.

[21] Nichols, M. D. and Padgett, W.J. (2006). A Bootstrap Control Chart for Weibull Per- centiles. Quality and Reliability Engineering International, 22, 141-151.

[22] Oluyede, B.O., Huang, S. and Pararai, M. (2014). A New Class of Generalized Dagum Distribution with Applications to Income and Lifetime Data. Journal of Statistical and Econometric Methods, $3(2), 125-151$.

[23] Oluyede, B. O. and Yang, T. (2015). A New Class of Generalized Lindley Distributions with Applications. Journal of Statistical Computation and Simulations, 85(10), 2072-2100.

[24] Paranaiba, P. F., Ortega, E. M. M., Cordeiro, G. M. and Pescim, R. R. (2011). The Beta Burr XII Distribution with Application to Lifetime Data. Computational Statistics and Data Analysis, 55, $1118-1136$.

[25] Pham, H. and Lai, C. D. (2007). On Recent Generalizations of the Weibull Distribution.IEEE, Transaction on Reliability, 56, 454-458.

[26] Proschan, F. (1963). Theoretical Explanation of Observed Decreasing Failure Rate. Tech- nometrics, 
$5,375-383$

[27] Rajarshi, S. and Rajarshi, M. B. (1988). Bathtub Distributions: A Review. Communications in Statistics-Theory and Methods, 17, 2521-2597.

[28] Rodrigues, R. N. (1977). A Guide to the Burr XII Distributions. Biometrika, 64, 129-134.

[29] Stuart, A. and Ord, K. (1994). Kendall's Advanced Theory of Statistics, Vol I: Distribution Theorem. 6th Edition, Oxford University Press, 1, 52.

[30] Tadikamalla, P. R. (1980) A Look at the Burr and Related Distributions. International Statistical Review, 48, 337-344. 


\section{Appendices}

\section{Appendix A. R Code: Deftne Functions}

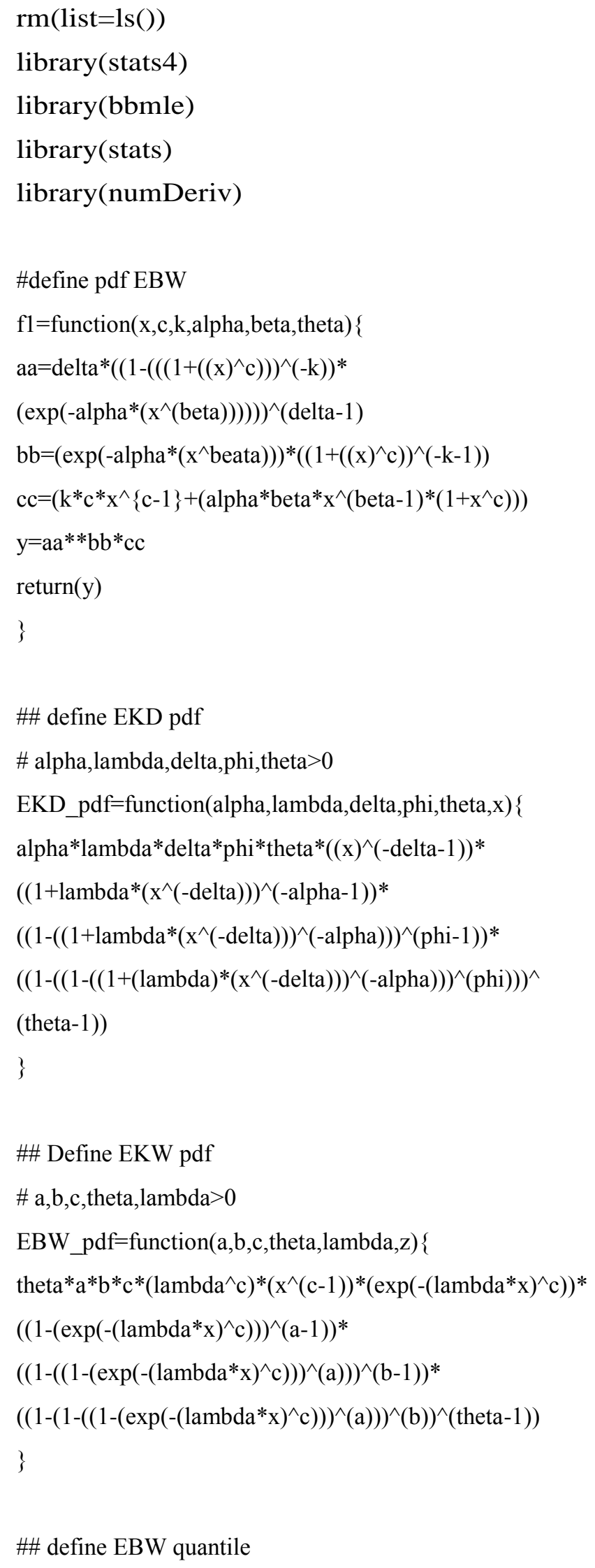




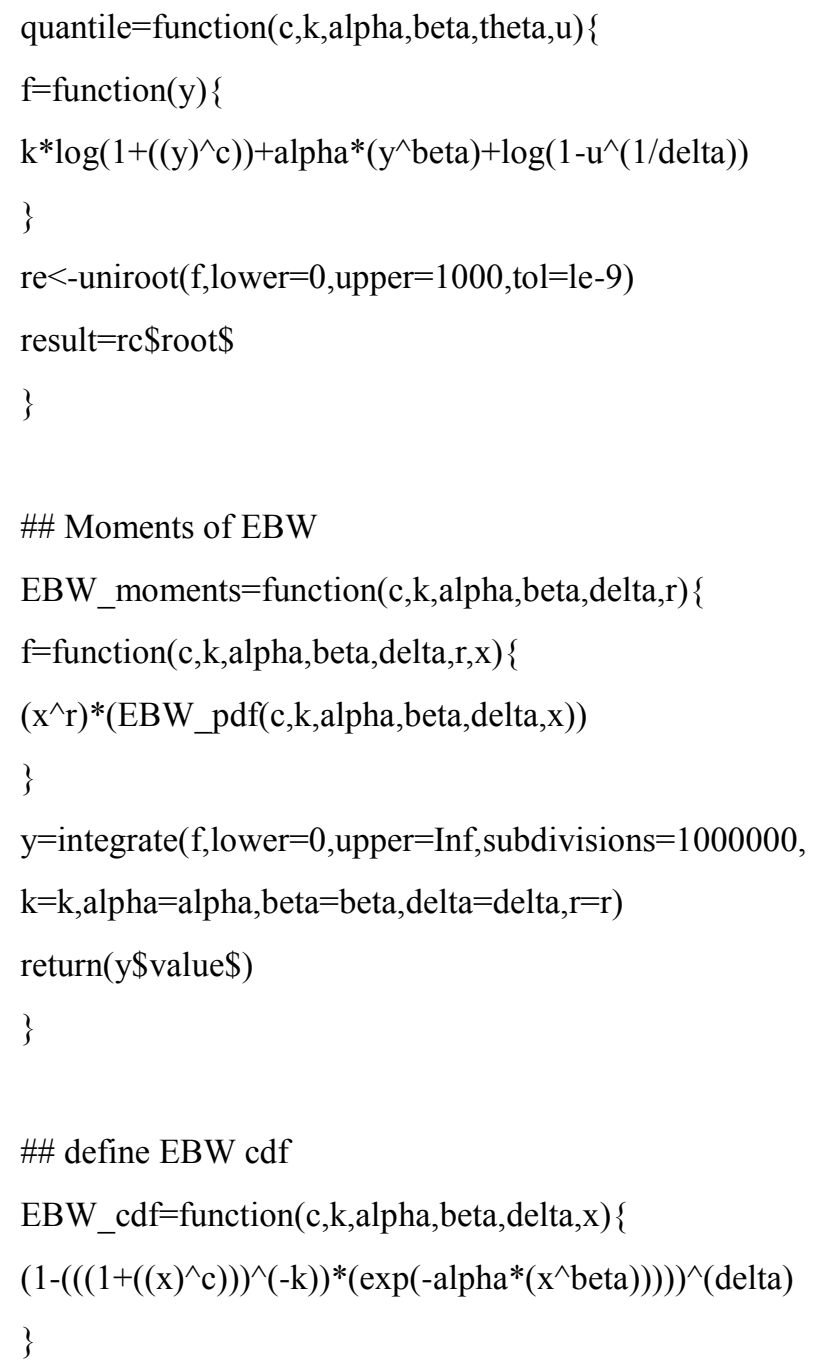




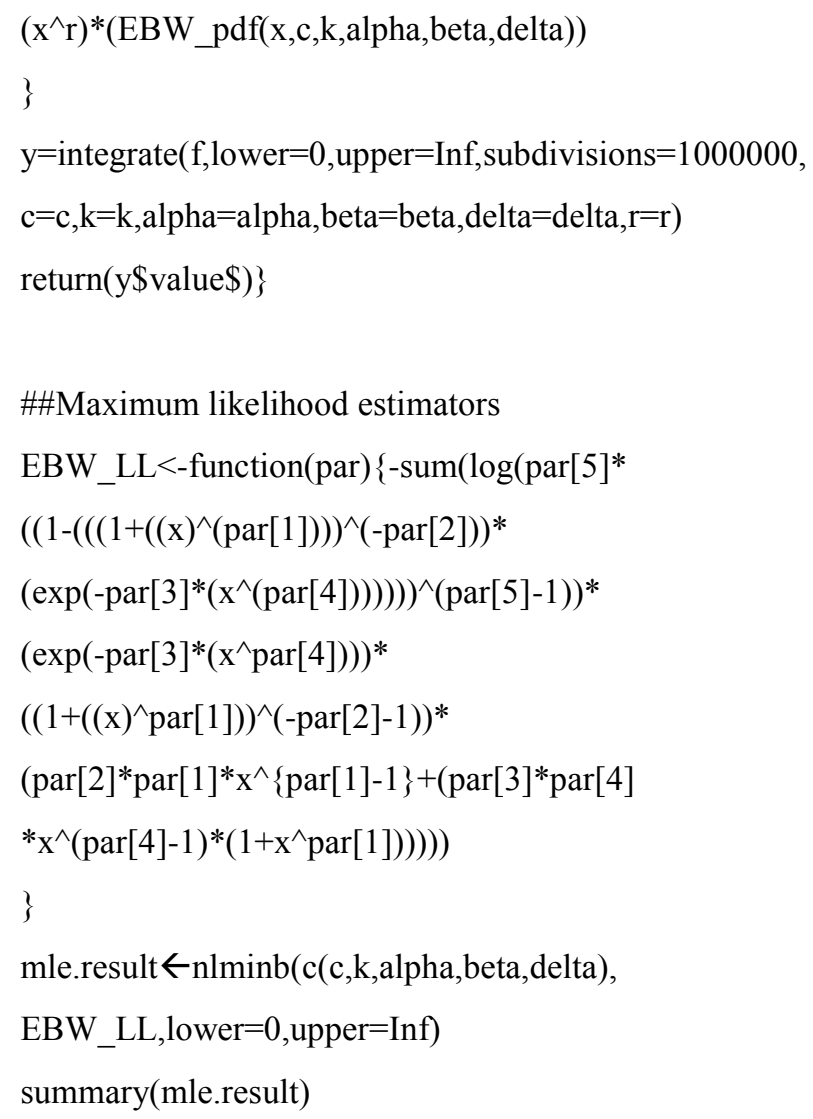


